\title{
Ferropicrite from the Lalibela area in the Ethiopian large igneous province
}

\author{
Minyahl Teferi Desta*, Dereje Ayalew ${ }^{* *}$, Akira Ishiwatari ${ }^{* *}$, Shoji Arai $^{\dagger}$ and Akihiro TAMURA ${ }^{\dagger}$ \\ *Department of Earth Science, Graduate School of Science, Tohoku University, Aoba, Sendai 980-8578, Japan \\ ${ }^{* *}$ School of Earth Sciences, Addis Ababa University, P.O.Box 1176, Addis Ababa, Ethiopia \\ ${ }^{* * *}$ Center for Northeast Asian Studies, Tohoku University, Kawauchi 41 Aoba, Sendai 980-8576, Japan \\ †Department of Earth Sciences, Faculty of Science, Kanazawa University, Kakuma-machi, Kanazawa 920-1192, Japan
}

\begin{abstract}
Ferropicrite $\left(\mathrm{FeO}^{*}=14.0 \mathrm{wt} \%\right.$ and $\left.\mathrm{MgO}=13.9 \mathrm{wt} \%\right)$ and picritic ferrobasalt $(\mathrm{FeO} *=14.7 \mathrm{wt} \%$ and $\mathrm{MgO}=$ $10.8 \mathrm{wt} \%$ ) lava flows are found near Lalibela in the Oligocene ( $30 \mathrm{Ma})$ Ethiopian large igneous province (LIP) in association with ultratitaniferous transitional basalt and picrite of the second high-Ti (HT2) series. The dominant phenocryst in the studied samples is Mg-rich olivine (up to Fo ${ }_{88.9}$ ) with high $\mathrm{CaO}$ contents (to 0.42 $\mathrm{wt} \%$ ) without any kink band structure, indicating that the olivines are crystallized from a magnesian melt. Spinel microphenocrysts and inclusions in olivine are characterized by extremely high $\mathrm{Cr} \#$ (79-84), moderate $\mathrm{Mg} \#$ (18-51), moderate $\mathrm{Fe}^{3+} \#(11-39)$ and high $\mathrm{TiO}_{2}$ (3.6-14.8 wt\%). The clinopyroxene phenocrysts are $\mathrm{Mg} \#=74-88, \mathrm{TiO}_{2}=0.84-1.82 \mathrm{wt} \%$, and $\mathrm{Al}_{2} \mathrm{O}_{3}=1.2-3.2 \mathrm{wt} \%$ in the cores. The REE contents of clinopyroxenes display enrichment in LREE $\left(\mathrm{La}_{N} / \mathrm{Yb}_{\mathrm{N}}=1.2-1.9\right)$ and MREE $\left(\mathrm{Eu}_{\mathrm{N}} / \mathrm{Yb}_{\mathrm{N}}=3.3-4.3\right)$ relative to HREE. Relative depletion of HFSE (e.g., $\mathrm{Nb}$ and $\mathrm{Zr}$ ) is also observed. In general, these trace element characteristics of clinopyroxene are similar to those of the HT2 basalts. Some of the clinopyroxene crystals show strong reverse zoning with abrupt increase in $\mathrm{Cr}, \mathrm{Ni}$ and $\mathrm{Mg} \#$ from core to rim, which may be resulted from reaction of melt with mantle peridotite during magma ascent. Bulk rock chemistry of the studied samples exhibits very low $\mathrm{Al}_{2} \mathrm{O}_{3} / \mathrm{TiO}_{2}$ (1.8-2.2) and high $\mathrm{Zr} / \mathrm{Y}$ (8.2-10.2) ratios. These may indicate the important role for garnet during melting and that the lavas were formed by a small degree of partial melting which apparently contradicts to the high $\mathrm{Cr} \#$ of spinel. In view of low $\mathrm{Cr} / \mathrm{Al}$ ratio of the bulk rock, the high $\mathrm{Cr} \#$ of spinel suggests very high temperature of the magma (and the mantle plume). The origin of these ferropicrite and picritic ferrobasalt could be attributed to high pressure partial melting of peridotite-eclogite (pyroxenite) mixture that possibly incorporated recycled oceanic crust components.
\end{abstract}

Keywords: Ferropicrite, Cr-spinel, Large igneous province, Oligocene, Lalibela, Eclogitic source

\section{INTRODUCTION}

Ferropicrites are subalkaline or mildly alkaline primitive rocks that were reported for the first time from the Paleoproterozoic Pechenga volcanic belt of the Kola Peninsula by Hanski and Smolkin (1989), who defined ferropicrite as an $\mathrm{MgO}$ rich volcanic rock with $\mathrm{FeO}^{*}$ higher than $14 \mathrm{wt} \%$. Ferropicrites may have similar $\mathrm{MgO}$ contents to komatiites $(\sim 19 \mathrm{wt} \%)$ but significantly higher $\mathrm{TiO}_{2}$ contents $(>1 \mathrm{wt} \%)$ like meimechite (Le Bas, 2000). The geochemical characteristics of ferropicrites and meimechites indicates their derivation by lower de-

doi:10.2465/jmps.131125b

M.T. Desta, mintfr09@gmail.com Corresponding author gree of partial melting at higher pressure as compared to common continental flood basalt picrite that originate as more homogenized and voluminous melting at lower pressures (Gibson, 2002). For the last two decades, a number of ferropicritic whole-rock compositions have been reported from the Precambrian volcanic belts e.g., Slave and Superior Provinces (Goldstein and Francis, 2008; Francis et al., 1999) and from the Phanerozoic continental flood basalt provinces (CFB) (Siberian Traps, Karoo, Paraná-Etendeka, North Atlantic, Emeishan; Wooden et al., 1993; Gibson et al., 2000; Riley et al., 2005; Zhang et al., 2006). Ichiyama et al. (2006) reported ferropicritic, highly magnesian olivine cumulates from a Permian accreted oceanic plateau, southwestern Japan. Recently, several petrogenetic models are provided for 


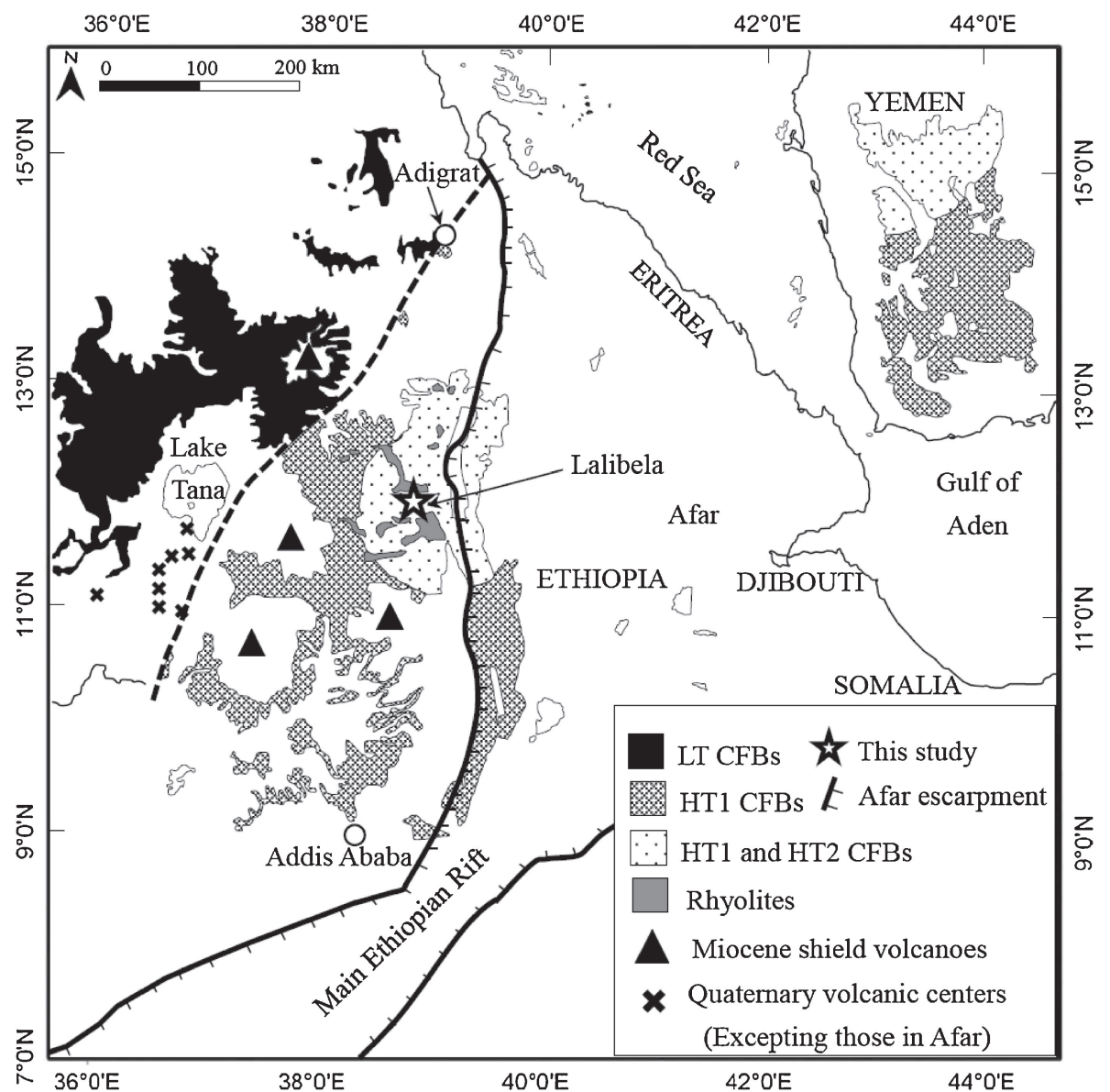

Figure 1. Sketch map of the Ethiopian large igneous province (LIP) including the Yemen conjugate margin, modified after Beccaluva et al. (2009) and Natali et al. (2011). Oligocene $(\sim 30 \mathrm{Ma})$ volcanic rocks: LT, Low-Ti tholeiitic basalts; HT1, High-Ti tholeiitic basalts; HT2, very High-Ti transitional basalts and picrites. Location of ferropicrite from the Dilb road section reported in this study is indicated by star. Miocene to Quaternary volcanic rocks in the Main Ethiopian Rift (MER), are not shown. CFB, Continental flood basalt

the origin of ferropicrite. Gibson (2002) describes the significance of recycled oceanic crust as a 're-fertilizer' of peridotite in the starting mantle plume head. Ichiyama et al. (2006) and Tuff et al. (2005) also suggested analogous models with recycled eclogitic or pyroxenitic mantle source, respectively. On the basis of silicate liquid immiscibility study, Jakobsen et al. (2005) interpreted that ferropicrites could also form by mixing of evolved, immiscible Fe-rich liquid with picritic mantle melts. More recently, Sobolev et al. (2007) described that iron-rich source regions in the mantle can be produced by pyroxenite veining. Generally, ferropicrites are attributed to anomalously hot mantle sources and mantle plumes (Hanski and Smolkin, 1995; Goldstein and Francis, 2008).

Picritic lavas from Ethiopian LIP are representative of the second high-Ti (HT2) series, as described by Pik et al. (1998) and Beccaluva et al. (2009) and are overlain by rhyolitic ignimbrite of 30 Ma age (Hofmann et al., 1997). In previous literatures the occurrence of $\mathrm{Fe}$-rich picrite in Ethiopian large igneous province (LIP) has been unrecognized.

In this study, we report the results of the mineral chemistry and whole rock geochemistry of ferropicrite
(Sample 1) and picritic ferrobasalt (Samples 2 and 3) rocks from the eastern part of Ethiopian large igneous province in order to obtain insight into the characteristics of their mantle source. Ferropicrite and picritic ferrobasalt samples were obtained from the Dilb road section of the Lalibela area, eastern portion of Ethiopian LIP (Fig. 1).

\section{GEOLOGICAL SETTING}

The Oligocene Ethiopian LIP represents one of the youngest, best exposed sequences of mafic and felsic volcanic rocks, associated with incipient lithospheric breakup (extensional tectonics) and plume impingement (Marty et al., 1996). It is dissected by the Main Ethiopian Rift (MER)-Red Sea-Gulf of Aden rift system (with the Afar $\mathrm{R}-\mathrm{R}-\mathrm{R}$-triple junction), which indicates the close association of magmatism and lithospheric extension and hence satisfy the plume theory. In addition, the tectonomagmatic and geophysical studies shows that the existence of deep mantle plume (Ritsema et al., 1999; Courtillot et al., 2003). The present remnants of Ethiopian flood volcanism cover an area of at least $6 \times 10^{6} \mathrm{~km}^{2}$ and have a total volume of about $\sim 3.5 \times 10^{5} \mathrm{~km}^{3}$ (Mohr and Zanet- 
tin, 1988). Rochette et al. (1998) estimated as the original volume reached up to $10^{6} \mathrm{~km}^{3}$. This voluminous basaltic magmatism was emplaced in a rather short time interval $30 \pm 1 \mathrm{Ma}$ (Hofmann et al., 1997) and successively followed by a number of huge shield volcanoes (Piccirillo et al., 1979). These shield volcanoes are a conspicuous feature of the Ethiopian LIP and distinguish it from other LIPs, such as Deccan and Karoo (Kieffer et al., 2004) and Siberia. The ferropicrite and picritic ferrobasalt of this study is considered as a part of the main flood basalt volcanism ( $30 \mathrm{Ma})$. Ayalew et al. (2002) and Ayalew and Ishiwatari (2011) described the presence of significant volume of rhyolite (up to $20 \%$ ) with a maximum thickness of $\sim 500 \mathrm{~m}$ mostly on the upper part of the Ethiopian continental flood basalt sequence. The final phase of volcanism (i.e., Pliocene to present) mostly concentrated along the Main Ethiopian Rift and Afar region. However, volumetrically small volcanic rocks as old as 45-40 Ma are found in southern and south-western Ethiopia (George et al., 1998). All the Ethiopian plateau rocks rest either directly over the Neoprotrozoic crystalline basement or over the Mesozoic sedimentary sequences. According to Pik et al. (1998) and Beccaluva et al. (2009), the Northern Ethiopian plateau basalts are classified into three magma types based on their $\mathrm{TiO}_{2}$ content (Fig. 1): Low-Ti tholeiites (LT) distributed in the northwestern areas; the first high-Ti lavas (HT1) in the eastern areas and ultratitaniferous transitional basalt and picrites of the second high-Ti (HT2) series, which are concentrated in the Lalibela area close to the Afar depression. The lava piles in the Lalibela area reaches up to $\sim 1800 \mathrm{~m}$ thick, consisting of basaltic and picritic units capped by rhyolitic ignimbrite. The HT2 picrites are geographically localized in the high-Ti sub-province and the ferropicrite and picritic ferrobasalt rocks occur associated with HT2 basalt and picrite series. Ferropicrites constitute volumetrically minor parts of Ethiopian LIP and they typically form thin flows at or near the base of thick lava piles in the Lalibela area.

\section{ANALYTICAL METHODS}

The major element analysis of the constituent minerals of these samples was determined by electron probe micro analyser (EPMA) using energy dispersive X-ray spectrometer Oxford Link ISIS equipped on the JEOL JSM-5410 Scanning electron microscope (SEM) at the Earth Science department laboratory of Tohoku University, Japan. The operating conditions were at $15 \mathrm{kV}$ acceleration voltage and a beam current of $1 \mathrm{nA}$. A number of natural standards were applied for calibration, and the data were corrected with ZAF quantitative procedure.
Major and some trace elements for the whole-rock analyses, such as V, Cr, Ni, Rb, Sr, Ba, Y, Zr, Nb, were carried out by X-ray fluorescence spectrometer (XRF-RIX 2100) at the aforementioned department, Tohoku University. Three glass beads were prepared by using fusion procedure, which consists of heating a 1:5 mixture of sample and flux $\left(\mathrm{Li}_{2} \mathrm{~B}_{4} \mathrm{O}_{7}\right)$ at $\sim 1050{ }^{\circ} \mathrm{C}$. The Loss on Ignition (LOI) is determined by weighing a 1 gram of rock powder before and after ignition at $750{ }^{\circ} \mathrm{C}$ for 6 hours. Trace element concentrations of clinopyroxene in ferropicrite and picritic ferrobasalts were determined by a laser ablation (193 nm ArF excimer: MicroLas GeoLas Q-plus)inductively coupled plasma spectrometer Agilent 7500S (LA-ICP-MS) at Kanazawa University (Ishida et al., 2004). The analysis was conducted by ablating spot of $50-60 \mu \mathrm{m}$ in diameter at laser power of $8 \mathrm{~J} / \mathrm{cm}^{2}$. NIST SRM 612 glass was used as a standard. The trace element concentration of NIST SRM 612 was selected from Pearce et al. (1997). ${ }^{29} \mathrm{Si}$ was used as internal standard for pyroxene, with concentration of $\mathrm{SiO}_{2}$ determined by EPMA.

\section{WHOLE ROCK CHEMISTRY}

\section{Major and trace elements}

Table 1 shows whole-rock analyses of the ferropicrite and picritic ferrobasalt from the Lalibela area. The studied samples are characterized by high $\mathrm{TiO}_{2}(3.8-5.1 \mathrm{wt} \%)$ and $\mathrm{FeO}^{*}$ (total $\mathrm{Fe}$ as $\left.\mathrm{FeO}\right)(14.0-14.7 \mathrm{wt} \%$ ) (Fig. 2). The $\mathrm{FeO}^{*}$ content is within the range of ferropicrites of other LIPs (Superior Province, Pechenga, Siberia, ParanáEtendeka and Japan). The Fe/Mn ratios of the bulk rock range from 78 to 82 , which is higher than those reported for the Ethiopian picritic lava (70-76; Rogers et al., 2010). The $\mathrm{Ni} / \mathrm{Cr}$ ratio is also high (0.4 in ferropicrite). The $\mathrm{MgO}$ content ranges from 10.5 to $13.8 \mathrm{wt} \%$ and Sample 1 satisfies the definition of picrite $(>12 \mathrm{wt} \%$ $\mathrm{MgO})$ (Le Bas, 2000). A general decrease of Ni (500 to $350 \mathrm{ppm}$ ) with decreasing $\mathrm{Mg \#} \mathrm{(64} \mathrm{to} \mathrm{56)} \mathrm{implies} \mathrm{re-}$ moval of olivine through evolution from ferropicritic to ferro-basaltic composition. They show enrichment of high field strength elements (HFSE), e.g., $\mathrm{P}_{2} \mathrm{O}_{5}(0.35-$ $0.64 \mathrm{wt} \%), \mathrm{Nb}(24-32 \mathrm{ppm}), \mathrm{Y}$ (34-39 ppm) and $\mathrm{TiO}_{2}$ (3.8-5.1 wt\%) and their increase with decreasing $\mathrm{MgO}$ (Figs. 2 and 3). They are further characterized by very high V (407-490 ppm) content. The content of Zr (283$378 \mathrm{ppm}$ ) in the Ethiopian ferropicrite is very high and is comparable to that of Siberian ferropicrite, but the latter is characterized by higher $\mathrm{Nb} / \mathrm{Y}$ ratio. Figure 4 shows the variations of $\mathrm{Al}_{2} \mathrm{O}_{3} / \mathrm{TiO}_{2}$ with respect to $\mathrm{Zr} / \mathrm{Y}$ ratios for the ferropicrite and picritic ferrobasalt. In comparison 
Table 1. Whole rock chemistry of the ferropicrite and picritic ferrobasalts from the Lalibela area, Ethiopian LIP

\begin{tabular}{|c|c|c|c|}
\hline \multirow{2}{*}{$\begin{array}{l}\text { Rock } \\
\text { Sample }\end{array}$} & \multirow{2}{*}{$\begin{array}{c}\text { Ferropicrite } \\
1\end{array}$} & \multicolumn{2}{|c|}{ Picritic ferrobasalts } \\
\hline & & 2 & 3 \\
\hline \multicolumn{4}{|c|}{ Major elements (wt\%) } \\
\hline $\mathrm{SiO}_{2}$ & 45.82 & 43.04 & 44.03 \\
\hline $\mathrm{TiO}_{2}$ & 3.84 & 4.74 & 5.04 \\
\hline $\mathrm{Al}_{2} \mathrm{O}_{3}$ & 8.58 & 8.65 & 9.23 \\
\hline $\mathrm{FeO}^{*}$ & 14.00 & 14.50 & 14.70 \\
\hline $\mathrm{CaO}$ & 10.00 & 9.87 & 10.63 \\
\hline $\mathrm{MgO}$ & 13.92 & 10.05 & 10.81 \\
\hline $\mathrm{MnO}$ & 0.18 & 0.18 & 0.18 \\
\hline $\mathrm{K}_{2} \mathrm{O}$ & 0.76 & 0.90 & 0.76 \\
\hline $\mathrm{Na}_{2} \mathrm{O}$ & 1.82 & 1.89 & 2.02 \\
\hline $\mathrm{P}_{2} \mathrm{O}_{5}$ & 0.35 & 0.62 & 0.58 \\
\hline Total & 99.27 & 94.44 & 97.98 \\
\hline LOI & -0.01 & 1.58 & 0.10 \\
\hline $\mathrm{Mg \#}$ & 63.9 & 55.3 & 56.7 \\
\hline \multicolumn{4}{|c|}{ Trace elements (ppm) } \\
\hline V & 407 & 463 & 490 \\
\hline $\mathrm{Cr}$ & 195 & 40.8 & 18.2 \\
\hline $\mathrm{Ni}$ & 501 & 389 & 356 \\
\hline $\mathrm{Rb}$ & 16.1 & 20.3 & 9.6 \\
\hline $\mathrm{Sr}$ & 415 & 503 & 567 \\
\hline $\mathrm{Ba}$ & 210 & 268 & 282 \\
\hline $\mathrm{Y}$ & 34.4 & 38.9 & 37.2 \\
\hline $\mathrm{Zr}$ & 283 & 359 & 378 \\
\hline $\mathrm{Nb}$ & 23.6 & 31.0 & 31.9 \\
\hline
\end{tabular}

*Total iron as $\mathrm{FeO}$.

$\mathrm{Mg} \#\left(=\mathrm{Mg} / \mathrm{Mg}+\mathrm{Fe}_{\text {Total }}\right) \times 100$.

LOI, Loss on ignition.

with other ferropicrites (Superior Province, Pechenga, Paraná-Etendeka and Japan) the Ethiopian ferropicrite and picritic ferrobasalt exhibit low $\mathrm{Al}_{2} \mathrm{O}_{3} / \mathrm{TiO}_{2}$ ratio close to Siberian ferropicrite $(\sim 2.2)$ and are similar to HT2 basalt and picrite (Beccaluva et al., 2009).

According to the Pik et al. (1998) classification of the Ethiopian LIP based on Ti/Y ratio, the studied samples falls in the HT1 compositional field (not shown). The high $\mathrm{FeO}^{*}$ content of these samples are similar to the first high-Ti basalts (HT1) of Adigrat area (Beccaluva et al., 2009). However, the latter is characterized by lower $\mathrm{MgO}(<10 \mathrm{wt} \%), \mathrm{CaO} / \mathrm{Al}_{2} \mathrm{O}_{3}(\sim 0.5)$ and $\mathrm{Ni}(\leq 110 \mathrm{ppm})$, supporting HT2 affiliation of our samples.

\section{PETROGRAPHY AND MINERAL CHEMISTRY}

Ferropicrite and picritic ferrobasalts examined in this study shows porphyritic texture with phenocryst phases of olivine (15-30 vol\%) and augitic clinopyroxene (10$25 \mathrm{vol} \%$ ) (Fig. 5). Minor plagioclase microphenocrysts occur in one of the studied samples. Chrome-spinels are included in olivine and rarely occur as microphenocrysts set in the groundmass, which comprises a mixture
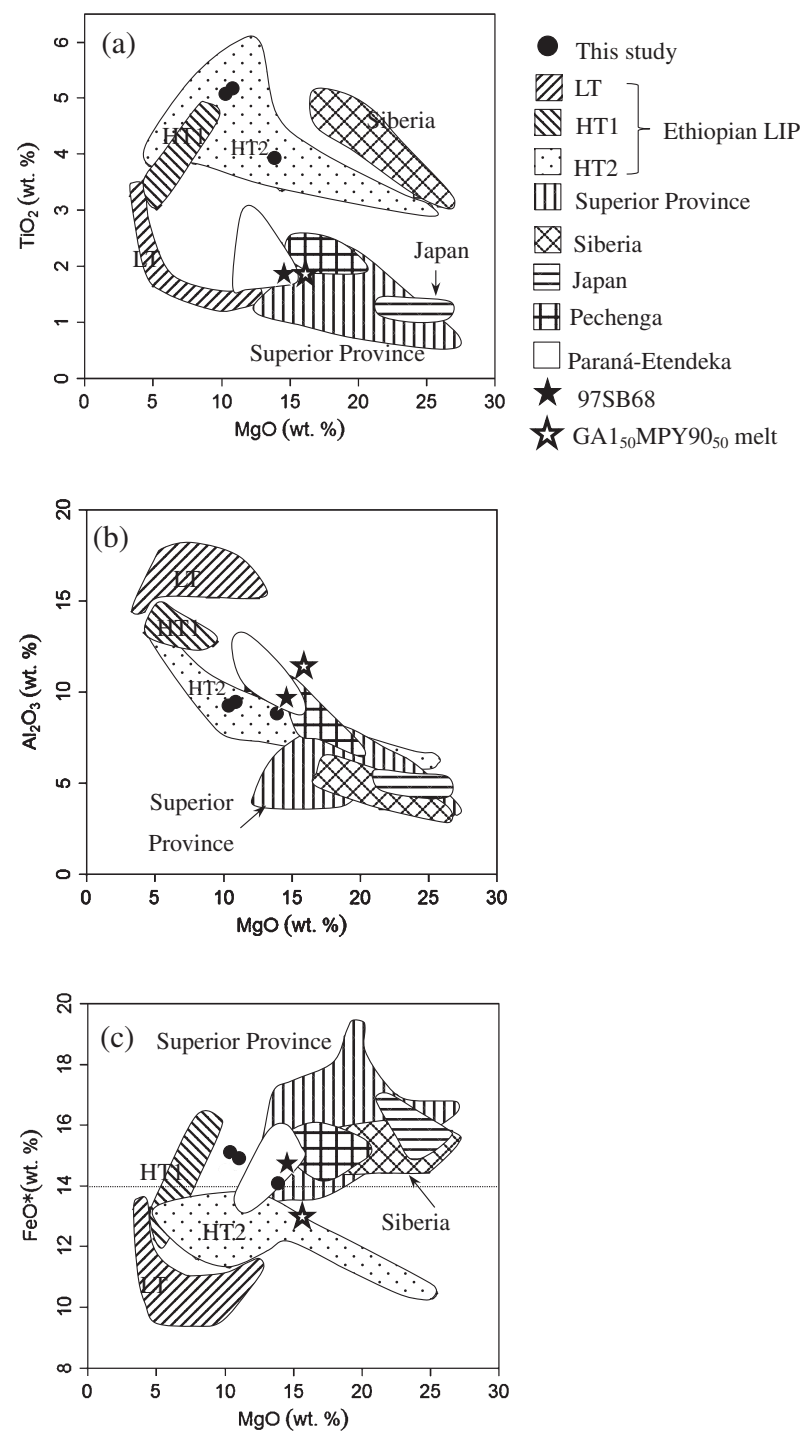

Figure 2. Major element variations with respect to $\mathrm{MgO}$ of the ferropicrite and picritic ferrobasalt rocks. For comparison, Ethiopian LIP (LT, low-Ti basalt; HT1, high-Ti 1 basalt; HT2, high-Ti 2 basalt and picrite; Beccaluva et al., 2009), ferropicrites from Superior Province (Goldstein and Francis, 2008), Pechenga (Hanski and Smolkin, 1995), Siberia (Arndt et al., 1995), Paraná-Etendeka (Gibson et al., 2000), Japan (Ichiyama et al., 2006), the composition of a picritic melt produced from $\mathrm{GA}_{50} \mathrm{MPY} 0_{50}$ at $3.5 \mathrm{GPa}$ (Yaxley and Green, 1998) and 97SB68 starting composition of Tuff et al. (2005) experiment.

of plagioclase, clinopyroxene, and ilmenite. Olivine phenocrysts typically show hexagonal euhedral shape (Figs. $5 \mathrm{a}$ and $5 \mathrm{~b}$ ) with irregular cracks and slight iddingsite alteration along the cracks. Some of the faces on olivine crystal are curved and embayed. Smaller olivines $(\sim 2$ $\mathrm{mm})$ are generally rounded and exhibit a rim of distinctive red tarnish of iddingsite formed by oxidation of olivine. Some olivine crystals are completely replaced by iddingsite. Deformed, kink-banded crystals are absent. 

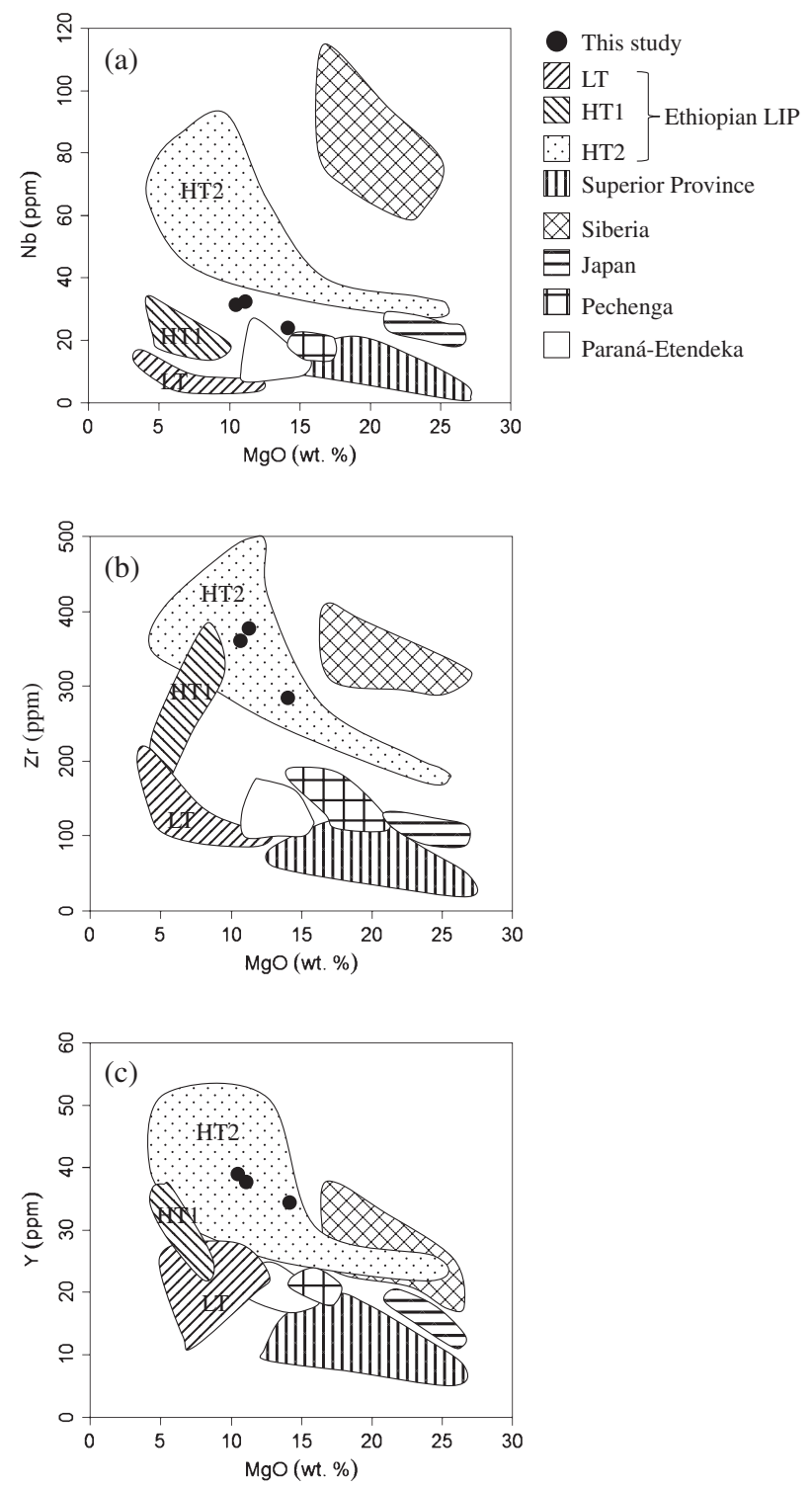

Figure 3. Trace element variation with respect to $\mathrm{MgO}$ of Ethiopian ferropicrite and picritic ferrobasalt. Symbols and data sources are as in Figure 2.

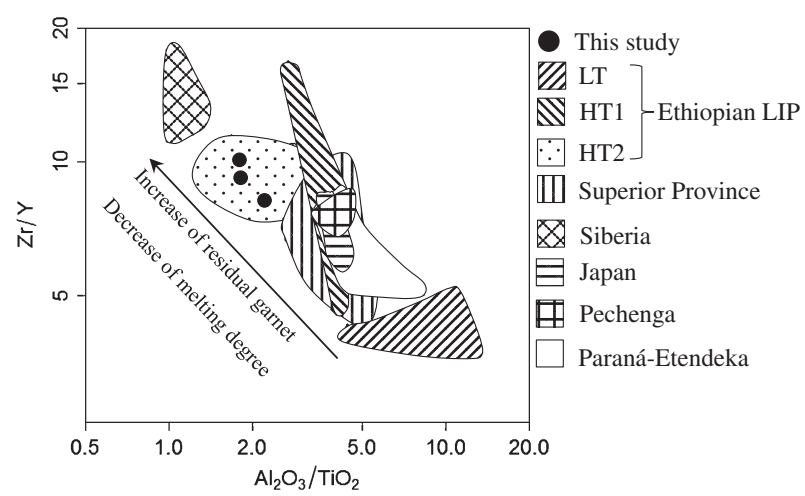

Figure 4. Bulk-rock $\mathrm{Al}_{2} \mathrm{O}_{3} / \mathrm{TiO}_{2}$ versus $\mathrm{Zr} / \mathrm{Y}$ plot for the Ethiopian ferropicrite and picritic ferrobasalt. Data sources are as in Figure 2. The arrow assumes a single peridotitic source.
Clinopyroxenes occur as euhedral, elongated pinkish phenocrysts $(<4 \mathrm{~mm})$ which are partially embayed and resorbed (Figs. 5c and 5d). In places, olivine and clinopyroxene occur together as glomerocrysts up to $7 \mathrm{~mm}$ in size in which olivines appear to be interstitial between clinopyroxenes. Ilmenites (up to $0.2 \mathrm{~mm}$ ) are also included in some clinopyroxene phenocrysts. Representative compositions of the main mineral phases are shown in Tables 2-5.

Olivine phenocrysts in the studied samples are homogenous and show a narrow compositional range ( $\left.\mathrm{Fo}_{78-88}\right)$, which corresponds to the previously reported values from HT2 basalts and picrites $\left(\mathrm{Fo}_{77-90}\right.$, Beccaluva et al., 2009). The Fo content of olivines from the Ethiopian ferropicrite and picritic ferrobasalt fall within the range of those in Archean and Phanerozoic ferropicrites (Fo $_{83-84}$ in Pechenga complex, Hanski and Smolkin,

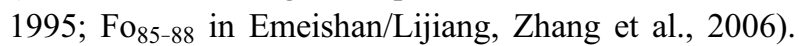
Generally, the largest olivines are the most magneisan, whereas smaller crystals are relatively $\mathrm{Fe}$-rich. $\mathrm{NiO}$ in the Fo-rich olivine cores reaches $0.3 \mathrm{wt} \%$ (Fig. 6). The $\mathrm{CaO}$ contents range from 0.28 to $0.42 \mathrm{wt} \%$, indicating that they are not mantle xenocrysts (Simkin and Smith, 1970; Gurenko et al., 1996; Thompson and Gibson, 2000; Kawabata et al., 2011) and MnO contents vary between 0.14 and $0.24 \mathrm{wt} \%$.

The $\mathrm{Cr}$-spinels are characterized by extremely high $\mathrm{Cr} \#[100 \mathrm{Cr} /(\mathrm{Cr}+\mathrm{Al})]$ (up to 84$)$, moderate $\mathrm{Mg} \#[100 \mathrm{Mg} /$ $\left.\left(\mathrm{Mg}+\mathrm{Fe}^{2+}\right)\right](18-51)$ and moderate $\mathrm{Fe}^{3+} \#\left[100 \mathrm{Fe}^{3+} /(\mathrm{Cr}+\right.$ $\left.\left.\mathrm{Al}+\mathrm{Fe}^{3+}\right)\right](11-39)($ Table 3$)$. The $\mathrm{Cr}_{2} \mathrm{O}_{3}$ content varies from 14 to $49 \mathrm{wt} \%$. The $\mathrm{TiO}_{2}$ contents of $\mathrm{Cr}-$ spinels are very high (3.6-14.8 wt\%) as compared to $\mathrm{Cr}$-spinels in meimechite and depleted ferropicrites from Vestfjella (Heinonen and Luttinen, 2010). The $\mathrm{Cr} \#$ of $\mathrm{Cr}$-spinels from these ferropicrite and picritic ferrobasalt is as high as those in boninite and is distinctly higher than $\mathrm{Cr}-$ spinels in abyssal peridotites (Dick and Bullen, 1984), MORB (Sigurdsson and Schilling, 1976), picritic basalts (e.g., West Greenland; Larsen and Pedersen, 2000, Emeishan; Kamenetsky et al., 2012, Iceland; Sigurdsson et al., 2000, Sorachi-Yezo; Ichiyama et al., 2012) and ferropicrites from Pechenga (Hanski, 1992) and accreted oceanic plateau in Japan (Mino-Tamba belt; Ichiyama et al., 2006), (Figs. 7a and 7b). Figure 8 shows the relationship between $\mathrm{Cr}$-spinel and bulk rock in their $\mathrm{Cr} / \mathrm{Al}$ ratios of the studied samples and other related rocks. The high $\mathrm{Cr} /$ $\mathrm{Al}$ of the $\mathrm{Cr}-$ spinels and very low $\mathrm{Cr} / \mathrm{Al}$ of the host rock is unique feature of the studied Ethiopian ferropicritic rocks.

Clinopyroxene phenocrysts are $\mathrm{Mg} \#=74-88, \mathrm{TiO}_{2}=$ 0.84-1.82 wt \%, and $\mathrm{Al}_{2} \mathrm{O}_{3}=1.2-3.2 \mathrm{wt} \%$ in the cores. Clinopyroxenes are $\mathrm{Mg}$-rich augite $\left(\mathrm{Wo}_{43-46} \mathrm{En}_{41-48} \mathrm{Fs}_{7-15}\right)$ in composition. They commonly show normal zoning 


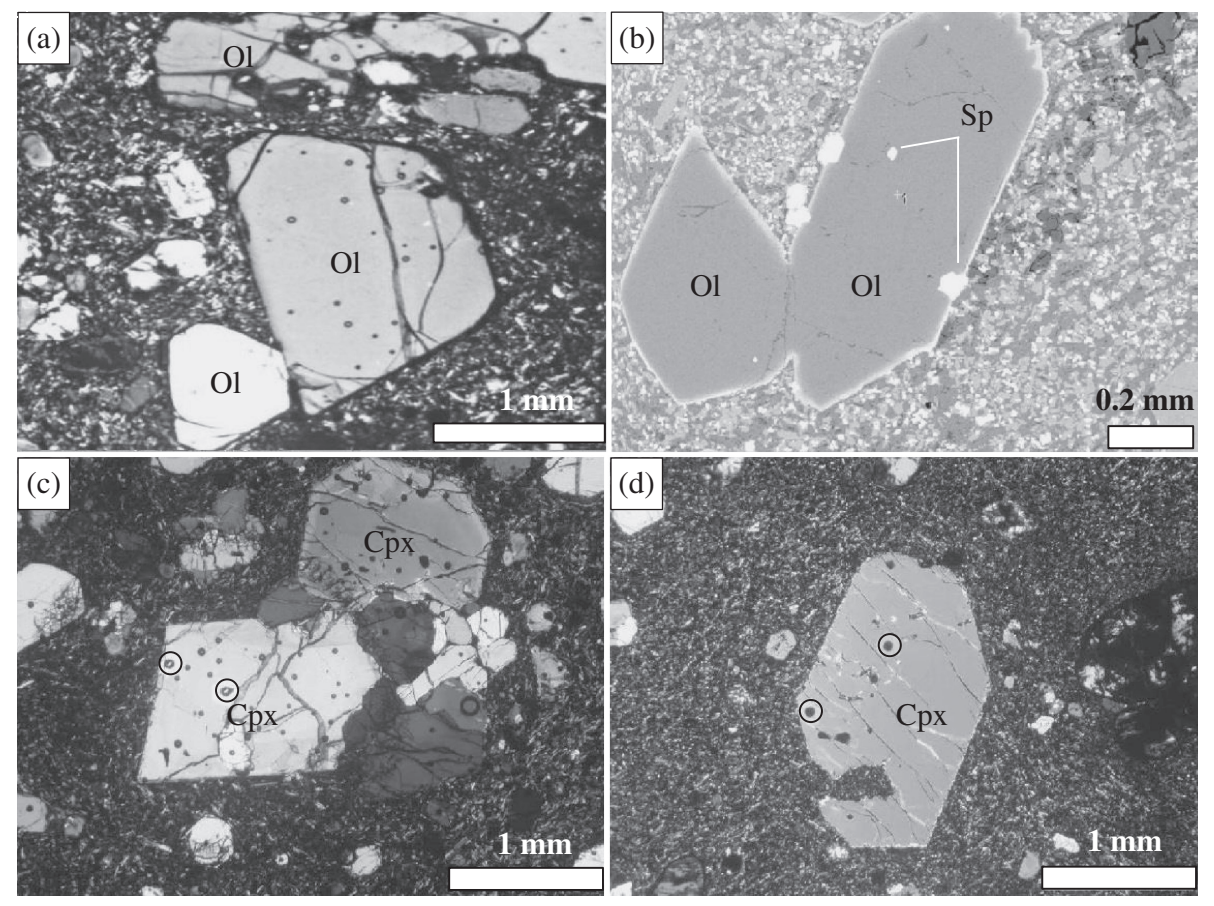

Figure 5. Photomicrographs of Ethiopian ferropicrite and picritic ferrobasalt. (a) Crossed polarized light showing porphyritic texture with abundant magnesian olivine phenocrysts. (b) Back-scattered electron images of olivine phenocrysts and spinel inclusions. Subhedral to euhedral olivine phenocrysts are set in a microcrystalline groundmass. (c) Crossed polarized light images of clinopyroxene with reverse zoning, Cpx3 of Sample 1 and (d) that with resorbed texture, $\mathrm{Cpx} 1$ of Sample 2. Small circles in the clinopyroxene phenocrysts show ICPMS analytical points (Table 5). Ol, olivine; $\mathrm{Cpx}$, clinopyroxene; $\mathrm{Sp}$, spinel.

Table 2. Representative analyses of olivine phenocrysts in ferropicrite and picritic ferrobasalt rocks from Lalibela area, Ethiopian LIP

\begin{tabular}{|c|c|c|c|c|c|c|c|c|c|c|c|c|c|c|c|}
\hline \multirow{3}{*}{$\begin{array}{l}\text { Sample } \\
\text { Mineral }\end{array}$} & \multicolumn{5}{|c|}{1 Ferropicrite } & \multicolumn{4}{|c|}{2 Picritic ferrobasalt } & \multicolumn{6}{|c|}{3 Picritic ferrobasalt } \\
\hline & \multirow{2}{*}{$\frac{\text { Ol1 }}{\text { Core }}$} & \multicolumn{2}{|c|}{$\mathrm{O} 12$} & \multicolumn{2}{|c|}{$\mathrm{O} 3$} & \multicolumn{2}{|c|}{$\mathrm{O} 13$} & \multicolumn{2}{|c|}{$\mathrm{O} 14$} & \multirow{2}{*}{$\begin{array}{c}\text { Ol2 } \\
\text { Core }\end{array}$} & \multicolumn{2}{|c|}{$\mathrm{Ol3}$} & \multirow{2}{*}{$\frac{\mathrm{O} 5}{\text { Core }}$} & \multicolumn{2}{|c|}{ O16 } \\
\hline & & Core & $\operatorname{Rim}$ & ore & $\operatorname{Rim}$ & Core & $\operatorname{Rim}$ & Core & Rin & & Core & Rim & & Core & $\operatorname{Rim}$ \\
\hline $\mathrm{iO}_{2}$ & 39.03 & 41.68 & 40.00 & .94 & & 42.12 & 40.73 & .01 & & 40.04 & 40.51 & 40.96 & 39.92 & 9.29 & 0.97 \\
\hline $\mathrm{O}^{*}$ & 9.48 & 12.39 & 16.33 & 11.41 & 18.59 & 10.63 & 11.91 & 10.52 & & 4.40 & & 4.52 & 13.23 & 6.48 & 17.26 \\
\hline & & & & & & & & & & & & & & & \\
\hline & 39.35 & 6.96 & 42.32 & & & & 46. & 47. & & & & 44.32 & & 40.87 & 42.70 \\
\hline & 29 & 34 & 0.32 & & & & 31 & & & & & 0.3 & & 0.37 & 0.36 \\
\hline & 27 & 0.22 & 0.25 & 0.25 & & & 0.44 & 0.30 & & 0.31 & 0.29 & 0.45 & 0.29 & 0.28 & 0.21 \\
\hline Total & 98.66 & 1.75 & 99.48 & 99.77 & 98 & & 99.76 & 99.43 & & 99.00 & 99.91 & 00.85 & 97.55 & 97.52 & 101.74 \\
\hline \multicolumn{16}{|c|}{ Cations $(\mathrm{O}=4)$} \\
\hline $\mathrm{Si}$ & 1.016 & 015 & 1.016 & 1.014 & & & 1.011 & 1.014 & & 1.014 & 1.018 & 1.017 & 1.02 & 1.020 & 1.01 \\
\hline $\mathrm{Fe}^{2+}$ & 24 & 0.252 & 0.347 & 0.236 & & & 0.247 & & & 0.3 & 0.3 & 0.301 & 0.28 & 0.358 & 0.35 \\
\hline & 05 & 0.003 & 0.006 & 0.004 & & & 0.004 & 0.003 & & 0.002 & 0.005 & 0.005 & 0.004 & 0.005 & 0.00 \\
\hline & & 1.704 & 1.602 & & & & 1.7 & & & 1.651 & 1.641 & 1.640 & 1.659 & 1.582 & 1.58 \\
\hline & 08 & 009 & 0.009 & 0.011 & & & 0.008 & 0.007 & & 0.011 & 0.010 & 0.010 & 0.009 & 0.010 & 0.01 \\
\hline & & 004 & 0.005 & & & & 0.009 & 0.006 & & 0.006 & 0.006 & 0.009 & 0.006 & 0.006 & 0.00 \\
\hline & 2.990 & 2.990 & 2.985 & 2.988 & 2.993 & 2.981 & 2.988 & 2.986 & 2.985 & 2.989 & 2.989 & 2.982 & 2.981 & 2.981 & 2.98 \\
\hline & 78.26 & 87.12 & 82.20 & 87.92 & 79.41 & 88.95 & 87.34 & 88.85 & 86.23 & 84.41 & 84.15 & 84.49 & 85.43 & 81.55 & 81.51 \\
\hline
\end{tabular}

* Total $\mathrm{Fe}$ as $\mathrm{FeO}$.

Abbreviation: Ol, olivine.

and it gives a trend of increasing $\mathrm{Fe}, \mathrm{Al}$, and $\mathrm{Ti}$ concentrations and decreasing $\mathrm{Mg} \#, \mathrm{Si}$ and $\mathrm{Cr}$ from core to rim (Fig. 9). However, some of the studied clinopyroxene crystals are very rich in $\mathrm{Cr}$ (up to $6240 \mathrm{ppm}$ ) and display very strong reverse zoning with significant increase of compatible elements (such as $\mathrm{Cr}$ and $\mathrm{Ni}$; Tables 4 and 5) and decreasing REEs from core to rim. This unusual, significant reverse zoning provides insight in to the origin of these lavas (see Section Eclogite versus peridotite as the source for Ethiopian ferropicrite). The trace element composition of clinopyroxenes of ferropicrite and picritic ferrobasalt rocks resembles those of HT2 basalt (Beccaluva et al., 2009), but they are enriched in total REE contents than those of iron-poor picrites. The REE contents of clinopyroxenes display enrichment in $\operatorname{LREE}\left(\mathrm{La}_{\mathrm{N}} / \mathrm{Yb}_{\mathrm{N}}=1.2-1.9\right)$ and $\operatorname{MREE}\left(\mathrm{Eu}_{\mathrm{N}} / \mathrm{Yb}_{\mathrm{N}}=3.3-\right.$ 4.3) relative to HREE. The primitive mantle-normalized pattern (Fig. 10) of clinopyroxene is characterized by low 
Table 3. Representative analyses of spinel inclusions in olivine phenocrysts

\begin{tabular}{|c|c|c|c|c|c|c|c|c|}
\hline \multirow{2}{*}{$\frac{\text { Sample }}{\text { Mineral }}$} & \multicolumn{2}{|c|}{1 Ferropicrite } & \multicolumn{4}{|c|}{2 Picritic ferrobasalt } & \multicolumn{2}{|c|}{3 Picritic ferrobasalt } \\
\hline & Spl1(Ol1) & Spl4(O13) & Spl1(Ol1) & $\mathrm{Spl2(O13)}$ & Spl3(O14) & Spl5 Gm & $\mathrm{Spl2(O12)}$ & Spl5(O15) \\
\hline $\mathrm{TiO}_{2}$ & 14.81 & 9.60 & 6.22 & 3.81 & 3.60 & 3.75 & 7.08 & 5.99 \\
\hline $\mathrm{Al}_{2} \mathrm{O}_{3}$ & 8.02 & 5.52 & 5.85 & 6.85 & 6.44 & 5.90 & 6.12 & 4.74 \\
\hline $\mathrm{Cr}_{2} \mathrm{O}_{3}$ & 13.97 & 38.50 & 37.38 & 48.93 & 47.31 & 43.21 & 34.44 & 37.85 \\
\hline $\mathrm{FeO}^{*}$ & 51.77 & 32.61 & 39.39 & 30.74 & 30.62 & 39.50 & 39.07 & 43.86 \\
\hline $\mathrm{MnO}$ & 0.34 & 0.24 & 0.16 & 0.19 & 0.17 & 0.32 & 0.16 & 0.26 \\
\hline $\mathrm{MgO}$ & 5.80 & 9.47 & 9.18 & 7.79 & 11.08 & 3.74 & 8.27 & 4.86 \\
\hline Total & 94.71 & 95.94 & 98.18 & 98.31 & 99.22 & 96.42 & 95.14 & 97.56 \\
\hline \multicolumn{9}{|c|}{ Cations $(\mathrm{O}=4)$} \\
\hline $\mathrm{Ti}$ & 0.398 & 0.253 & 0.160 & 0.099 & 0.090 & 0.102 & 0.188 & 0.161 \\
\hline $\mathrm{Al}$ & 0.338 & 0.228 & 0.235 & 0.278 & 0.253 & 0.252 & 0.255 & 0.199 \\
\hline $\mathrm{Cr}$ & 0.395 & 1.065 & 1.009 & 1.332 & 1.247 & 1.238 & 0.962 & 1.067 \\
\hline $\mathrm{Fe}^{3+}$ & 0.470 & 0.202 & 0.437 & 0.192 & 0.319 & 0.306 & 0.407 & 0.413 \\
\hline $\mathrm{Fe}^{2+}$ & 1.079 & 0.752 & 0.688 & 0.693 & 0.535 & 0.890 & 0.748 & 0.894 \\
\hline $\mathrm{Mn}$ & 0.010 & 0.007 & 0.005 & 0.006 & 0.005 & 0.010 & 0.005 & 0.008 \\
\hline $\mathrm{Mg}$ & 0.309 & 0.494 & 0.467 & 0.400 & 0.551 & 0.202 & 0.436 & 0.258 \\
\hline Total & 2.999 & 3.001 & 3.001 & 3.000 & 3.000 & 3.000 & 3.001 & 3.000 \\
\hline Cr\# & 53.89 & 82.39 & 81.08 & 82.73 & 83.13 & 83.09 & 79.06 & 84.27 \\
\hline $\mathrm{Mg \#}$ & 22.28 & 39.65 & 40.44 & 36.59 & 50.74 & 18.49 & 36.81 & 22.40 \\
\hline $\mathrm{Fe}^{3+} \#$ & 39.06 & 13.54 & 25.97 & 10.66 & 17.54 & 17.05 & 25.05 & 24.60 \\
\hline
\end{tabular}

${ }^{*}$ Total $\mathrm{Fe}$ as $\mathrm{FeO}$.

$\mathrm{Cr} \#=100 \mathrm{Cr} /(\mathrm{Cr}+\mathrm{Al})$.

$\mathrm{Mg} \#=100 \mathrm{Mg} /\left(\mathrm{Mg}+\mathrm{Fe}^{2+}\right)$.

$\mathrm{Fe}^{3+} \#=100 \mathrm{Fe}^{3+} /\left(\mathrm{Cr}+\mathrm{Al}+\mathrm{Fe}^{3+}\right)$.

Abbreviations: Spl, spinel; Ol, olivine; Gm, groundmass.

Number of host olivine is indicated in parentheses.

Table 4. Representative analyses of clinopyroxene phenocrysts and microphenocrysts of ferropicrite and picritic ferrobasalt rocks from Ethiopian LIP

\begin{tabular}{|c|c|c|c|c|c|c|c|c|c|c|c|c|c|c|c|}
\hline \multirow{2}{*}{$\frac{\text { Sample }}{\text { Mineral }}$} & \multicolumn{4}{|c|}{1 Ferropicrite } & \multicolumn{5}{|c|}{2 Picritic ferrobasalt } & \multicolumn{6}{|c|}{3 Picritic ferrobasalt } \\
\hline & \multicolumn{2}{|c|}{ Cpx1 } & \multicolumn{2}{|c|}{$\mathrm{Cpx} 3$} & \multicolumn{2}{|c|}{ Cpx1 } & \multirow{2}{*}{$\frac{\text { Cpx1Gm }}{\text { Core }}$} & \multicolumn{2}{|c|}{ n $\quad \mathrm{Cpx} 3$} & \multicolumn{2}{|c|}{ Cpx1 } & \multicolumn{2}{|c|}{$\mathrm{Cpx} 2$} & \multicolumn{2}{|c|}{ Cpx3 } \\
\hline & Core & Rim & & Rim & Core & $\operatorname{Rim}$ & & Core & Rim & ore & $\overline{\operatorname{Rim}}$ & Core & Rim & Core & Rim \\
\hline $\mathrm{SiO}_{2}$ & 53 & & & & & 50.62 & 49.55 & 53.50 & & & & & & 50.81 & 48.08 \\
\hline & 0.84 & 1.68 & & & & 1.77 & & 1.08 & 1.80 & 1.34 & 2.21 & & 1.77 & 1.68 & 2.39 \\
\hline & 1.25 & 50 & & 2.74 & 2.55 & 2.62 & 2.82 & 1.22 & 2.76 & 1.75 & 3.49 & 3.21 & 3.18 & 2.98 & 4.14 \\
\hline $\mathrm{r}_{2} \mathrm{O}_{3}$ & 0.73 & 0.38 & 0.22 & 0.62 & 0.12 & 0.25 & 0.15 & 0.67 & 0.20 & 0.73 & 0.62 & 0.89 & 0.24 & 0.08 & 0.06 \\
\hline & 4.37 & 7.13 & 7.65 & 6.02 & 7.97 & 7.08 & 9.24 & 4.83 & 8.05 & 5.03 & 6.80 & 6.23 & 7.16 & 6.96 & 8.39 \\
\hline & & 0.20 & & 0.14 & 0.16 & 0.13 & & n.d. & n.d. & 0.15 & 0.17 & & 0.13 & 0.21 & 0.14 \\
\hline & & 15.33 & & 16.15 & 15.26 & 15.12 & & 16.96 & 15.33 & 15.97 & 14.54 & & 14.91 & 15.35 & 13.53 \\
\hline & & 20.96 & & & & 1.26 & & 22.6 & 20.73 & & & & & 1.26 & 21.06 \\
\hline Total & & 8.63 & & & & 8.85 & 98.73 & 100.86 & 10065 & & 98.19 & & & 9.33 & 0770 \\
\hline \multicolumn{16}{|c|}{ Cations $(\mathrm{O}=6)$} \\
\hline & & 00 & & & & & & & & & & & & & \\
\hline & & & & & & & & 0.0 & & & & & & & 0.0 \\
\hline & & 1 & & & & 0.1 & & 0.0 & & & & & & 31 & 0.1 \\
\hline & & 011 & & & & 0.007 & 0.004 & 0.019 & 0.0 & 0.022 & & 26 & 0.007 & 0.002 & 0.00 \\
\hline & & 224 & & & & 0.222 & 0.293 & 0.147 & 0.2 & & & & & 0.217 & 0.26 \\
\hline & & & & & & 0.00 & & n.d. & & & & & & .007 & 0.00 \\
\hline & & & & & & 0.84 & & 0.919 & 0.8 & & & & & .852 & 0.7 \\
\hline & & 844 & & & & & & 0.880 & & & & & & .848 & 0.86 \\
\hline Total & & 3.997 & & & & 3.992 & 4.006 & 3.991 & 3.985 & 3.991 & 3.999 & 3.992 & 3.994 & 3.997 & 4.000 \\
\hline Wo & & 43.8 & & & & & & 45.2 & 42.9 & & & & 44.8 & 44.2 & 45.4 \\
\hline $\mathrm{E}$ & & 44.6 & & & & 44.0 & & 47.2 & 44.1 & 46.4 & 43.0 & & 43.5 & 44.4 & 40.5 \\
\hline Fs & & 11.6 & & & & 11.6 & & 7.5 & 13.0 & 8.2 & 11.3 & 10.1 & 11.7 & 11.3 & 14.1 \\
\hline $\mathrm{Mg \#}$ & 87.63 & 79.31 & 77.78 & 82.71 & 77.34 & 79.2 & 74.33 & 86.22 & 77.25 & 84.98 & 79.22 & 81.67 & 78.78 & 79.72 & 74.19 \\
\hline
\end{tabular}

*Total $\mathrm{Fe}$ as $\mathrm{FeO}$.

$\mathrm{Mg} \#=100 \mathrm{Mg} /\left(\mathrm{Mg}+\mathrm{Fe}^{2+}\right)$.

Abbreviations: Cpx, clinopyroxene; Wo, wollastonite; En, enstatite; Fs, ferrosilite; Gm, groundmass; n.d., not detected. 
Table 5. Representative trace element analyses (LA-ICP-MS; values in ppm) of clinopyroxenes from ferropicrite and picritic ferrobasalt rocks from Lalibela area, Ethiopian LIP

\begin{tabular}{|c|c|c|c|c|c|c|c|c|c|c|}
\hline \multirow{3}{*}{$\begin{array}{l}\text { Sample } \\
\text { Mineral } \\
\end{array}$} & \multirow{2}{*}{\multicolumn{2}{|c|}{$\begin{array}{c}1 \text { Ferropicrite } \\
\mathrm{Cpx} 3 \\
\end{array}$}} & \multicolumn{4}{|c|}{2 Picritic ferrobasalt } & \multicolumn{4}{|c|}{3 Picritic ferrobasalt } \\
\hline & & & \multicolumn{2}{|c|}{ Cpx1 } & \multicolumn{2}{|c|}{$\mathrm{Cpx} 2$} & \multicolumn{2}{|c|}{ Cpx1 } & \multicolumn{2}{|c|}{$\mathrm{Cpx} 2$} \\
\hline & Core & Rim & Core & $\operatorname{Rim}$ & Core & $\operatorname{Rim}$ & Core & $\operatorname{Rim}$ & Core & Rim \\
\hline$\overline{\mathrm{Sc}}$ & 64.3 & 62.2 & 64.2 & 66.1 & 81.1 & 73.1 & 68.4 & 68.4 & 58.9 & 61.1 \\
\hline $\mathrm{Ti}$ & 7480 & 7840 & 10050 & 10010 & 11140 & 10600 & 8580 & 8690 & 7110 & 9750 \\
\hline V & 449 & 313 & 471 & 419 & 391 & 402 & 247 & 252 & 202 & 314 \\
\hline $\mathrm{Cr}$ & 886 & 4240 & 809 & 2040 & 2550 & 2440 & 6240 & 5850 & 2120 & 2320 \\
\hline Co & 52.6 & 44.9 & 49.0 & 49.3 & 47.4 & 48.4 & 39.2 & 39.1 & 41.7 & 42.0 \\
\hline $\mathrm{Ni}$ & 262 & 358 & 255 & 305 & 300 & 295 & 374 & 347 & 375 & 364 \\
\hline $\mathrm{Rb}$ & 0.004 & 0.043 & 0.02 & 0.10 & 0.01 & 0.01 & 0.01 & 0.001 & 0.004 & 0.01 \\
\hline $\mathrm{Sr}$ & 49.9 & 46.9 & 73.5 & 70.0 & 68.3 & 65.7 & 58.6 & 62.1 & 56.1 & 77.7 \\
\hline$Y$ & 14.6 & 11.2 & 18.0 & 15.5 & 14.2 & 16.8 & 11.0 & 11.3 & 10.6 & 14.1 \\
\hline $\mathrm{Zr}$ & 35.0 & 25.7 & 62.4 & 54.4 & 53.5 & 60.7 & 30.2 & 31.8 & 24.0 & 45.9 \\
\hline $\mathrm{Nb}$ & 0.1 & 0.2 & 0.3 & 0.3 & 0.3 & 0.3 & 0.2 & 0.2 & 0.1 & 0.2 \\
\hline Cs & n.d. & 0.002 & n.d. & 0.010 & n.d. & 0.0003 & n.d. & 0.002 & n.d. & n.d. \\
\hline $\mathrm{Ba}$ & 0.02 & 0.64 & 0.23 & 0.33 & 0.03 & 0.05 & 0.02 & 0.03 & 0.01 & 0.02 \\
\hline $\mathrm{La}$ & 2.2 & 1.4 & 3.3 & 2.8 & 2.7 & 2.8 & 2.1 & 2.3 & 1.6 & 2.6 \\
\hline $\mathrm{Ce}$ & 10.7 & 7.2 & 15.9 & 13.7 & 12.6 & 13.5 & 9.3 & 10.4 & 7.6 & 12.8 \\
\hline Pr & 2.1 & 1.4 & 2.9 & 2.6 & 2.4 & 2.5 & 1.7 & 1.9 & 1.5 & 2.4 \\
\hline $\mathrm{Nd}$ & 12.2 & 8.3 & 17.5 & 15.0 & 13.9 & 15.0 & 10.0 & 11.0 & 8.8 & 13.9 \\
\hline $\mathrm{Sm}$ & 4.0 & 2.8 & 5.5 & 4.8 & 4.6 & 5.1 & 3.3 & 3.6 & 3.0 & 4.5 \\
\hline $\mathrm{Eu}$ & 1.3 & 0.9 & 1.8 & 1.6 & 1.5 & 1.6 & 1.1 & 1.2 & 1.0 & 1.5 \\
\hline Gd & 4.3 & 3.1 & 5.7 & 4.9 & 4.6 & 5.2 & 3.5 & 3.8 & 3.3 & 4.5 \\
\hline $\mathrm{Tb}$ & 0.6 & 0.5 & 0.8 & 0.7 & 0.6 & 0.7 & 0.5 & 0.6 & 0.5 & 0.7 \\
\hline Dy & 3.6 & 2.7 & 4.5 & 3.9 & 3.6 & 4.0 & 2.9 & 3.1 & 2.7 & 3.7 \\
\hline Ho & 0.6 & 0.5 & 0.8 & 0.7 & 0.6 & 0.7 & 0.5 & 0.5 & 0.4 & 0.6 \\
\hline $\mathrm{Er}$ & 1.5 & 1.1 & 1.8 & 1.5 & 1.4 & 1.6 & 1.2 & 1.2 & 1.1 & 1.5 \\
\hline $\mathrm{Tm}$ & 0.2 & 0.1 & 0.2 & 0.2 & 0.2 & 0.2 & 0.1 & 0.2 & 0.1 & 0.2 \\
\hline $\mathrm{Yb}$ & 1.1 & 0.8 & 1.3 & 1.0 & 0.9 & 1.1 & 0.8 & 0.9 & 0.8 & 1.0 \\
\hline $\mathrm{Lu}$ & 0.1 & 0.1 & 0.2 & 0.1 & 0.1 & 0.1 & 0.1 & 0.1 & 0.1 & 0.1 \\
\hline $\mathrm{Hf}$ & 1.7 & 1.2 & 2.8 & 2.4 & 2.6 & 2.8 & 1.7 & 1.7 & 1.2 & 2.2 \\
\hline $\mathrm{Ta}$ & 0.02 & 0.02 & 0.05 & 0.04 & 0.04 & 0.04 & 0.03 & 0.03 & 0.01 & 0.04 \\
\hline $\mathrm{Pb}$ & 0.05 & 0.05 & 0.10 & 0.06 & 0.04 & 0.03 & 0.05 & 0.05 & 0.05 & 0.04 \\
\hline Th & 0.01 & 0.01 & 0.03 & 0.02 & 0.03 & 0.02 & 0.02 & 0.02 & 0.01 & 0.02 \\
\hline $\mathrm{U}$ & 0.003 & 0.003 & 0.007 & 0.004 & 0.004 & 0.003 & 0.002 & 0.006 & 0.002 & 0.005 \\
\hline
\end{tabular}

Abbreviations: Cpx, clinopyroxene; n.d. not detected.

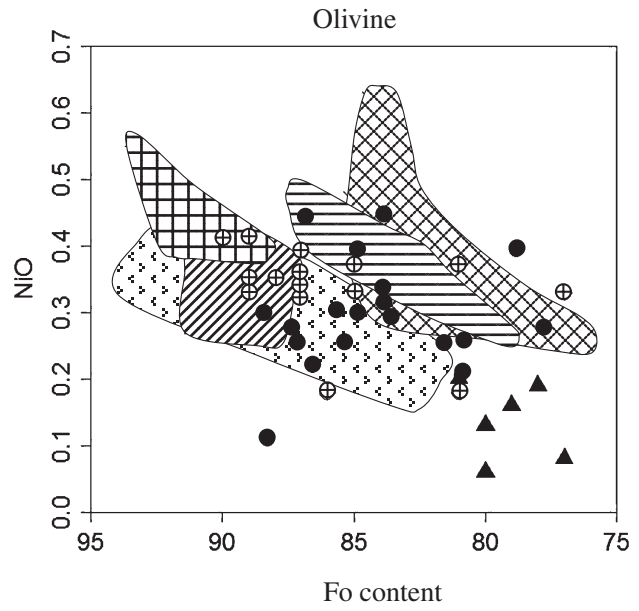

Figure 6. Fo content versus $\mathrm{NiO}(\mathrm{wt} \%)$ for olivines in ferropicrite and picritic ferrobasalt rocks from Ethiopian LIP. Those of the komatiites from Superior Province (Arndt et al., 1977); meimechite from Siberian traps (Elkins-Tanton et al., 2007), ferropicrites from Karoo and Ferrar provinces (Heinonen and Luttinen, 2008), Paraná-Etendeka provinces (Gibson et al., 2000) and picrites from Sorachi-Yezo (Ichiyama et al., 2012). The composition of olivines from the LT (low-Ti) and HT2 (high-Ti 2 basalt and picrites) of Ethiopian LIP (Beccaluva et al., 2009) are also shown for comparison. contents of $\mathrm{Pb}, \mathrm{Zr}$ and LILE (e.g., $\mathrm{Ba}, \mathrm{Rb}, \mathrm{Sr}$ ) relative to the neighboring elements, which is consistent with the low $D_{\text {LILE }}^{C p x / l i q}$ (Tuff and Gibson, 2007). Slight depletion of HFSE (e.g., $\mathrm{Nb}$ and $\mathrm{Ta}$ ) is also observed relative to LREE ( $\mathrm{La}$ and $\mathrm{Ce}$ ). There is no significant negative $\mathrm{Eu}$ anomaly $\left(\mathrm{Eu} / \mathrm{Eu}^{*}=0.95-1\right)$. 


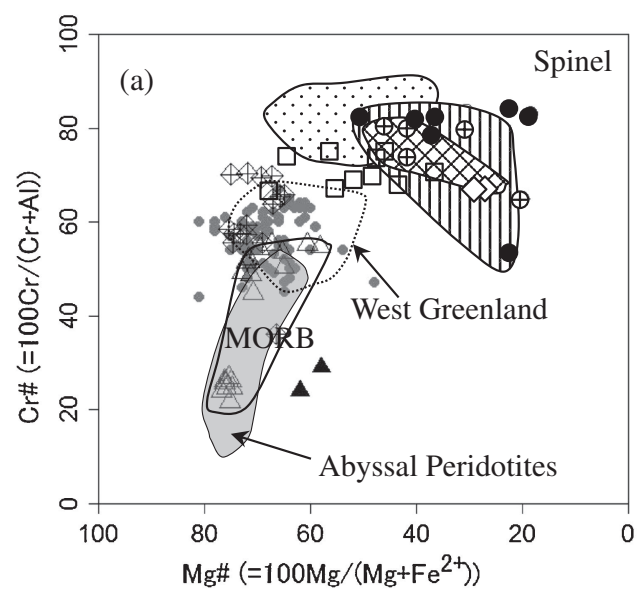

This study
$\triangle$ LT basalts
$\oplus$ HT2 picrite
Sorachi-Yezo
$\diamond$ Iceland
$\diamond$ Tamba ferropicrite
$\square$ Vestfjella meimechites \& ferropicrite
$\triangle$ MORB
سI Emeishan
$\because$ Boninite
$\bigotimes$ Pechenga

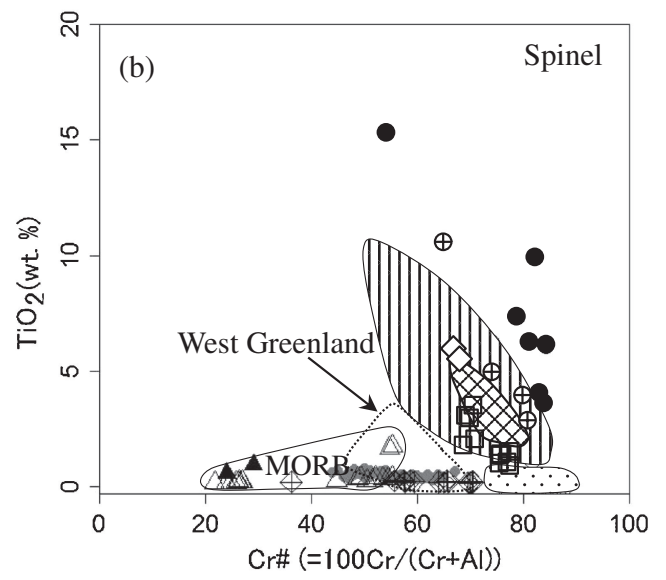

Figure 7. Cr-spinel composition of the Ethiopian ferropicrite and low-Ti basalt and HT2 picrites (Beccaluva et al., 2009). Those of the picrites from SorachiYezo (Ichiyama et al., 2012), Iceland (Sigurdsson et al., 2000), Tamba belt (Ichiyama et al., 2006), Emeishan (Kamenetsky et al., 2012), West Greenland (Larsen and Pedersen, 2000), Vestfjella meimechites and ferropicrite (Heinonen and Luttinen, 2010) and the ferropicrites from Pechenga (Hanski, 1992). The composition of MORB (Sigurdsson and Schilling, 1976), abyssal peridotites (Dick and Bullen, 1984) and boninite are also taken for comparison.

Plagioclase microphenocrysts have labradorite composition $\left(\mathrm{An}_{62} \mathrm{Ab}_{36} \mathrm{Or}_{2}\right)$ while plagioclase in groundmass is andesine $\left(\mathrm{An}_{41} \mathrm{Ab}_{54} \mathrm{Or}_{4}\right)$, consistent with its late crystallization. The opaque minerals in the groundmass are mainly ilmenite.

\section{DISCUSSION}

\section{Olivine Fo and $\mathrm{NiO}$ showing primitive mantle-derived melt}

Primitive rocks that are associated with continental flood basalt LIPs are scarce, but their presence provides important information on the parental sources of these voluminous mantle derived magma. The most $\mathrm{MgO}$-rich olivine that we analyzed is $\mathrm{Fo}_{88.9}$ (Table 2), which is higher than those of Paraná-Etendeka ferropicrite $\mathrm{Fo}_{85}$ (Gibson et al., 2000) and slightly lower than those of anhydrous fertile lherzolite $\left(\mathrm{Fo}_{90}\right)$ (e.g., Ishiwatari, 1985). They are only slightly less forsteritic than the most magnesian olivine from HT2 picrites $\left(\mathrm{Fo}_{90}\right)$ of Ethiopia (Beccaluva et al., 2009) and picrites from Karoo (Fo, ${ }_{90}$, Cox and Jamieson,
1974). More magnesian olivines from Hokkaido, Japan (Fo94, Ichiyama et al., 2012) and West Greenland (Fo92, Pedersen, 1985) may represent higher potential temperature in the mantle. The magnesian and high $\mathrm{NiO}$ (up to $0.3 \mathrm{wt} \%$ ) characteristics of olivine (Fig. 6) support the primitive, mantle-derived nature of the Ethiopian ferropicrite.

\section{Primary bulk-rock nature of the melt}

To assess the hypothetical primary melt compositions for the Ethiopian ferropicrite, we have tested a modeling program (PRIMELT2) (Herzberg and Asimow, 2008); however the outcome is unreliable due to the possible pyroxenite source component suggested by PRIMELT2 model. We thus focused on the modeling of primary melts on the basis of olivine-liquid equilibrium (Putirka, 2008). The analyzed samples are characterized by primitive olivine ( $>\mathrm{Fo}_{78}$; Table 2). The most $\mathrm{Mg}$-rich olivine compositions ( $\mathrm{Fo}_{88}$ in ferropicrite and $\mathrm{Fo}_{88-85}$ in picritic ferrobasalts) correspond to olivine that would crystallize from mantle derived melts. Assuming a $\mathrm{K}_{\mathrm{D}}(\mathrm{Fe}-\mathrm{Mg})^{\mathrm{ol}-\mathrm{liq}}$ of $0.32(\mathrm{Pu}-$ 


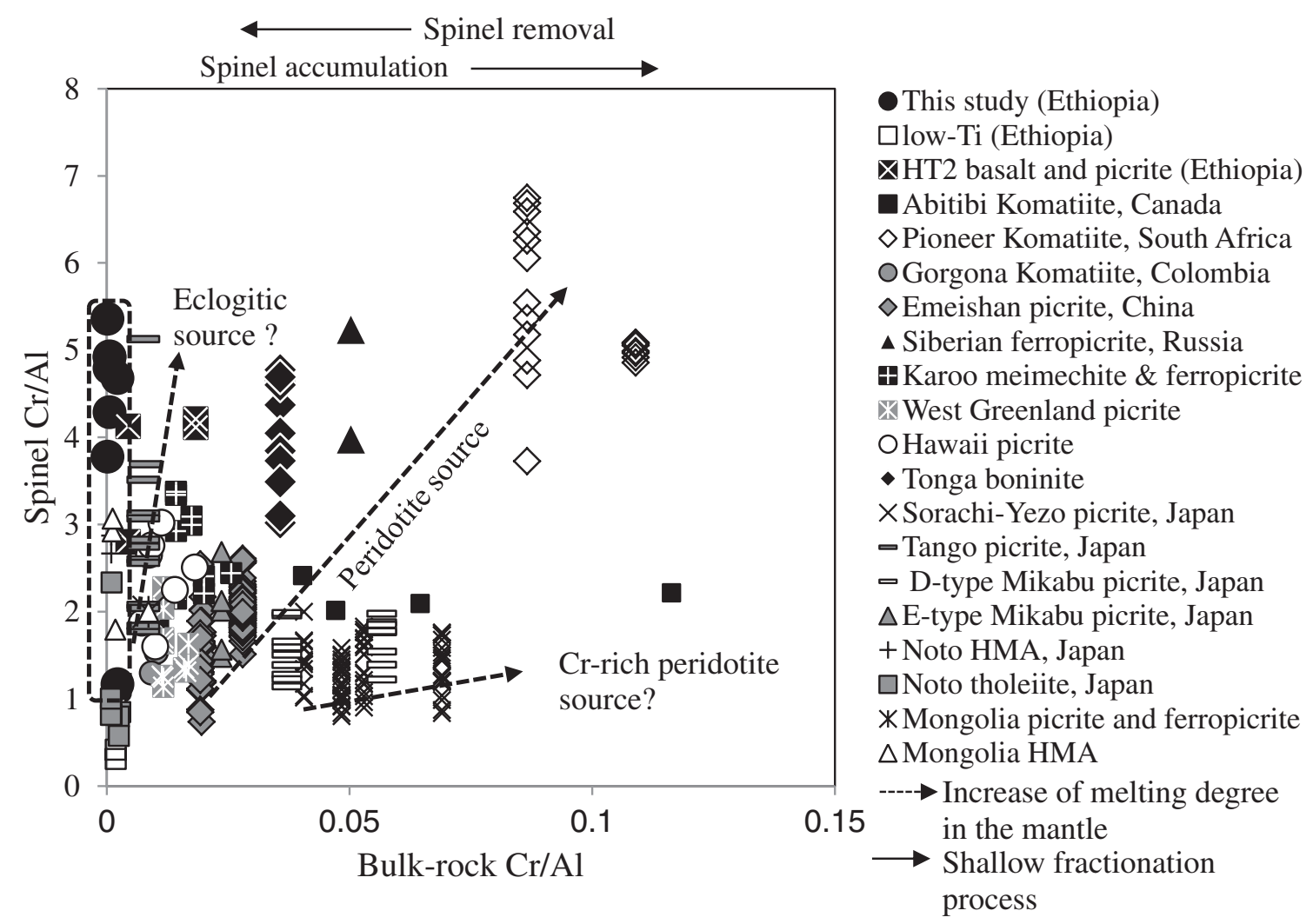

Figure 8. Bulk rock $\mathrm{Cr} / \mathrm{Al}$ versus Spinel $\mathrm{Cr} / \mathrm{Al}$ ratios for the Ethiopian ferropicrite and picritic ferrobasalts. Dashed arrows indicate the possible eclogitic and peridotitic sources. Data sources: Ethiopian LIP low-Ti and HT2 suits (Beccaluva et al., 2009); Abitibi komatiite (Barnes, 1985); Pioneer komatiite (Stiegler et al., 2012); Gorgona komatiite (Dietrich et al., 1981); Emeishan picrites (Kamenetsky et al., 2012); Siberian ferropicrite (Arndt et al., 1995); Karoo meimechite and ferropicrite (Heinonen and Luttinen, 2008, 2010); West Greenland picrite (Larsen and Pedersen, 2000); Hawaii picrite (Wilkinson and Hensel 1988); Tonga boninite (Sobolev and Danyushevsky, 1994); Sorachi-Yezo picrite (Ichiyama et al., 2012); Tango picrite (Ishiwatari and Imasaka, 2002); depleted (D-type) and enriched (E-type) picrites from Mikabu belt (Ichiyama et al., 2014); high magnesian andesite (HMA) and tholeiite lava Noto Peninsula (López and Ishiwatari, 2002); Mongolian high magnesian andesite and picrite (Erdenesaihan et al., 2013).

tirka, 2005), Fo $85-88$ would have been in equilibrium with a liquid with Mg\# 64-71 and 15-19 wt\% MgO. These observations indicate that the Ethiopian ferropicrite ( $\mathrm{Mg} \#$ 64), $14 \mathrm{wt} \% \mathrm{MgO}$ and $14 \mathrm{wt} \% \mathrm{FeO}^{*}$; Table 1 is likely to represent true Fe-rich mantle melt. Therefore, the high $\mathrm{FeO}^{*}$ content of Ethiopian ferropicrite is not an accumulation effect.

\section{Temperature estimates}

Gibson (2002) suggested that the ferropicritic liquid is generated by the partial melting of basalt plus peridotite mixture at $>1450{ }^{\circ} \mathrm{C}$ and $>4.5 \mathrm{GPa}$. The experimental result of Tuff et al. (2005) confirmed that a ferropicrite melts $100 \%$ at $5 \mathrm{GPa}$ and $1650{ }^{\circ} \mathrm{C}$, which is similar to the solidus ( $5 \mathrm{GPa}$ and $1625^{\circ} \mathrm{C}$ ) of silica-undersaturated garnet pyroxenite studied by Kogiso et al. (2003). These temperature are higher than the ambient upper mantle $\left(\mathrm{T}_{\mathrm{p}} \sim 1280-1475{ }^{\circ} \mathrm{C}\right.$; McKenzie and Bickle, 1988) and supports the derivation of ferropicrites from high temperature magma (and the plume). However, the available thermodynamic models are only compatible with peridotite sources (e.g., Herzberg and Asimow, 2008) and may not be applicable to estimate the physical conditions (pressure, mantle potential temperature) for pyroxenerich sources. Considering the compositional similarity between the Ethiopian ferropicrite and 97SB68 in terms of $\mathrm{MgO}, \mathrm{FeO}^{*}$ and $\mathrm{Al}_{2} \mathrm{O}_{3}$ (Fig. 2), the melting condition of our ferropicrite may be close to that of the melting experiment conducted by Tuff et al. (2005) as noted above.

Application of olivine-spinel geothermometer (Fabriès, 1979) to the ferropicrite and picritic ferrobasalt rocks results in temperatures as high as $1090{ }^{\circ} \mathrm{C}$ for the most chromian spinel. This may represent closure temperature of Fe-Mg exchange between spinel inclusion and host olivine during cooling of the magma, and hence gives the lower limit of the magmatic temperature estimate. 

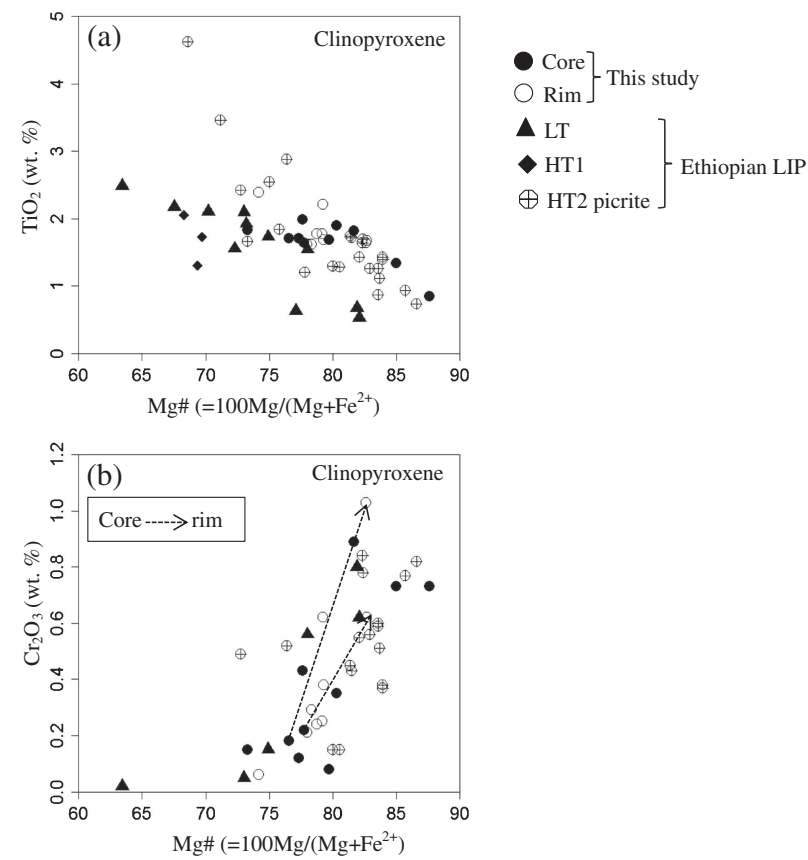

Figure 9. Plots of $\mathrm{Mg} \#$ versus $\mathrm{TiO}_{2}$ (a) and $\mathrm{Cr}_{2} \mathrm{O}_{3}$ (b) for the ferropicrite and picritic ferrobasalt of Ethiopian LIP. For comparison, clinopyroxenes from the high-Ti 2 picrite (Beccaluva et al., 2009) are also plotted. Dashed arrow represents the core to rim reverse zoning trend within a single crystal.

In addition, the $\mathrm{Cr}$ content of spinel may provide a clue for the temperature of the magma from which it crystallized. The $\mathrm{Cr}$ content of the melt in equilibrium with spinel (Cr solubility) increases with increasing temperature (Barnes, 1986). Effect of oxygen fugacity also matters, but co-existing ilmenite and absence of magnetite suggest low oxygen fugacity. The high $\mathrm{Cr} / \mathrm{Al}$ spinel would have been expected to crystallize from the magma having high $\mathrm{Cr} / \mathrm{Al}$ ratio, or the magmatic temperature was very high, and these in turn may indicate high degrees of melting, however, the bulk rock data of our samples shows very low content of Cr. Thus, it is likely that the observed high $\mathrm{Cr} / \mathrm{Al}$ ratio of spinel is due to high magmatic temperature.

\section{High degree melting of the source mantle?}

$\mathrm{Cr}$-spinel is one of the liquidus phases in basaltic magma and its chemical variation may have significant inferences about the degree of melting and source regions of magma (Dick and Bullen, 1984; Arai, 1994). The Cr-spinel of Ethiopian ferropicrite is significantly higher in $\mathrm{Cr} \#$ (average 78.7) than those of MORB, Emeishan high-Ti picrite (Kamenetsky et al., 2012), Pechenga ferropicrite (Hanski, 1992) and comparable to those of boninite (Fig. 7). The $\mathrm{TiO}_{2}$ content of Cr-spinel is also very high (Fig. 7b), which is consistent with the high $\mathrm{Ti}$ characteristics of bulk rock chemistry of the studied samples. Moreover, the $\mathrm{TiO}_{2}$ content of spinel is equal to or higher than those of LIP lavas (Kamenetsky et al., 2001). The high Cr/Al ratio of spinels from the Ethiopian ferropicrite and picritic ferrobasalts (Fig. 8) might indicate high degree of melting, but the bulk rock $\mathrm{Cr} / \mathrm{Al}$ ratio is very low and is not proportional to that of spinel. Thus we propose very low $\mathrm{Cr} / \mathrm{Al}$ of the source material (see Section $\mathbf{C r}$ / Al relationship between spinel and bulk rock: indicator of source lithology). Removal of spinel in the early stage of magma evolution is another possibility (Fig. 8), but is unlikely in view of the very primitive nature of the magma. However, this interpretation apparently contradicts to the suggested small degree of partial melting (of peridotite source) on the basis of low $\mathrm{Al}_{2} \mathrm{O}_{3} / \mathrm{TiO}_{2}$ and high $\mathrm{Zr} / \mathrm{Y}$ ratios of bulk rock compositions (Fig. 4).

$\mathrm{Cr} / \mathrm{Al}$ relationship between spinel and bulk rock: indicator of source lithology

Figure 8 shows the relationship between the bulk-rock $\mathrm{Cr} / \mathrm{Al}$ and spinel $\mathrm{Cr} / \mathrm{Al}$ ratios of the Ethiopian ferropicrite in comparison with mafic-ultramafic rocks of other igneous provinces. Based on this, the $\mathrm{Cr} / \mathrm{Al}$ ratio of spinels of Ethiopian ferropicrite is similar to those of Archean komatiite (Stiegler et al., 2012), Siberian ferropicrite (Arndt et al., 1995), and boninite (Sobolev and Danyushevsky, 1994), although the bulk-rock $\mathrm{Cr} / \mathrm{Al}$ ratio $(<0.005)$ is significantly lower than those mentioned above.

The observed very low bulk-rock $\mathrm{Cr} / \mathrm{Al}$ ratios in the Ethiopian ferropicrite and picritic ferrobasalts might be attributed to the removal of $\mathrm{Cr}$-spinel in the early stage of magma evolution. However, primitive nature of the studied samples does not support extensive early stage crystal fractionation. On the other hand, Sorachi-Yezo picrites (Ichiyama et al., 2012), Mikabu D-type picrites (Ichiyama et al., 2014) and Abitibi komatiite (Barnes, 1985) shows high bulk rock $\mathrm{Cr} / \mathrm{Al}$ but low spinel $\mathrm{Cr} / \mathrm{Al}$ ratios, which might indicate the accumulation of spinel in the magma (Fig. 8). However, occurrence of high Fo olivines in these rocks is compatible with high degree melting of peridotitic mantle rather than olivine accumulation process in shallow magma chamber. Thus, bulk rock $\mathrm{Cr}$ / $\mathrm{Al}$ ratio of the primitive magmas may be mainly controlled by lithology of the source material in the mantle. For example, if the source is eclogite (or pyroxenite), bulk rock $\mathrm{Cr} / \mathrm{Al}$ ratios of magma may not exceed 0.01 , but if the source is peridotite, $\mathrm{Cr} / \mathrm{Al}$ of the magma may be as high as 0.1 (Fig. 8). The very low $\mathrm{Cr} / \mathrm{Al}$ ratio of the Ethiopian ferropicrite together with the others of low bulk-rock $\mathrm{Cr} / \mathrm{Al}$ (such as those of Noto tholeiite and high 

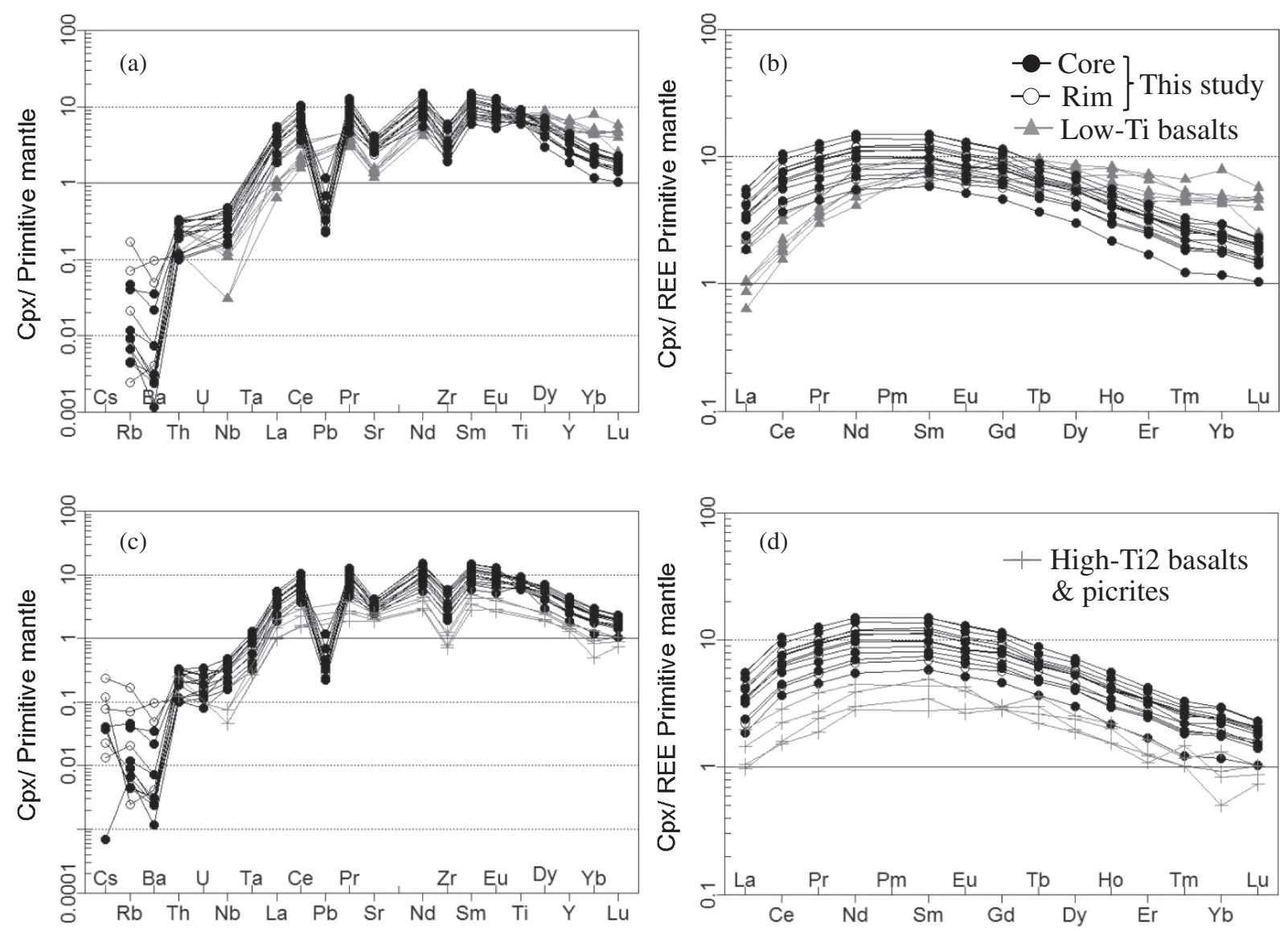

Figure 10. Primitive-mantle-normalized REE and trace element patterns for clinopyroxenes of ferropicrite and picritic ferrobasalt rocks from Lalibela area, Ethiopian LIP. Normalizing values are from McDonough and Sun (1995). For comparison, clinopyroxene compositions from low-Ti (a)-(b) and High-Ti 2 basalt and picrites (c)-(d) of Ethiopian LIP are also plotted after Beccaluva et al. (2009).

magnesian andesite (HMA), López and Ishiwatari, 2002; Mongolia picrite and HMA, Erdenesaihan et al., 2013; Hawaii picrite, Wilkinson and Hensel, 1988) (Fig. 8) may suggest eclogitic source, which is supported by the lines of evidence as discussed later (see Section Eclogite versus peridotite as the source for Ethiopian ferropicrite).

\section{High-pressure melting of the source mantle}

The increasing $\mathrm{Zr} / \mathrm{Y}$ and decreasing $\mathrm{Al}_{2} \mathrm{O}_{3} / \mathrm{TiO}_{2}$ ratios (Fig. 4) are generally attributed to increase of melting pressure or melt segregation depth and increasing stability of garnet in the residue (Walter, 1998). The Ethiopian ferropicrite and picritic ferrobasalts are characterized by higher $\mathrm{Zr} / \mathrm{Y}$ and lower $\mathrm{Al}_{2} \mathrm{O}_{3} / \mathrm{TiO}_{2}$ ratios than other ferropicritic rocks (e.g., Superior Province, Pechenga, Paraná-Etendeka and Mino-Tamba), indicating that their source region contained residual garnet and thus the partial melting took place in the garnet stability field. The low $\mathrm{Al}$ content in magmas such as ferropicrite (Tuff et al.,
2005) and Al-depleted komatiites (Arndt et al., 2008; Robin-Popieul et al., 2012) is interpreted to indicate the presence of residual garnet in the mantle source. Compared to the Ethiopian ferropicrite, the ferropicrites of Siberia exhibit higher $\mathrm{Zr} / \mathrm{Y}$ and lower $\mathrm{Al}_{2} \mathrm{O}_{3} / \mathrm{TiO}_{2}$ ratios, suggesting higher melting pressure.

The high Ni content (501 ppm) along with the low $\mathrm{Al}_{2} \mathrm{O}_{3}(\sim 9 \mathrm{wt} \%)$ of Ethiopian ferropicrite may also indicate that garnet was a residual phase in its mantle source. This interpretation is consistent with the high pressure experimental result on the Paraná-Etendeka ferropicrite produced by Tuff et al. (2005).

In addition, REE patterns of clinopyroxene phenocrysts from the studied samples show distinct convex upward patterns in chondrite-normalized diagrams (Fig. 10) with $\mathrm{La}_{\mathrm{N}} / \mathrm{Yb}_{\mathrm{N}}(1.2-1.9)$ and $\mathrm{Eu}_{\mathrm{N}} / \mathrm{Yb}_{\mathrm{N}}$ (3.3-4.3), resembling those reported HT2 basalt and picrites of Ethiopia (Beccaluva et al., 2009). Therefore, the strong HREE fractionation supports the presence of garnet in the mantle source region. 


\section{Bulk-rock high $\mathrm{Fe} / \mathrm{Mn}$ and $\mathrm{Ni} / \mathrm{Cr}$ ratios: a core-man- tle issue?}

High $\mathrm{Fe} / \mathrm{Mn}$ ratios of basalts could be resulted from coremantle interaction. However, on the basis of high $\mathrm{Fe} / \mathrm{Mn}$ and unradiogenic ${ }^{187} \mathrm{Os} /{ }^{188} \mathrm{Os}$ content of the Ethiopian picrite, Rogers et al. (2010) speculated that the entrapment of core material in the source of the Afar mantle plume is unlikely. Basaltic melts with high $\mathrm{Fe} / \mathrm{Mn}$ ( $>60)$ ratio can be formed by partial melting of pyroxenite at the degree of melting less than $70 \%$ (Kogiso and Hirschmann, 2001, Pertermann and Hirschmann, 2003, Kogiso et al., 2004) or of hydrous peridotite at the degree of melting greater than 50\% (Parman and Grove, 2004).

High fraction of clinopyroxene crystallization $(>30 \%$ ) could also produce a melt with high $\mathrm{Fe} / \mathrm{Mn}$ ratio (Liu et al., 2008). In view of high $\mathrm{MgO}(>10 \mathrm{wt} \%$ ) of the studied samples, such a high degree of crystallization is unrealistic. High Fe/Mn could be also resulted from the presence of residual garnet in the source (Herzberg, 2011), because $D_{M n}^{\text {Garnet/liquid }}$ is $\sim 7$ at low melt fraction (Pertermann and Hirschmann, 2003). High Ni content (500 ppm) and high $\mathrm{Ni} / \mathrm{Cr}$ ratio of the Ethiopian ferropicrite (Table 1) may also suggest contribution of $\mathrm{Fe}-\mathrm{Ni}$ metal in its magma genesis, but to attribute this to the core-mantle reaction must be too speculative.

\section{Eclogite versus peridotite as the source for Ethiopian ferropicrite}

The genesis of ferropicritic rocks have been considered to be associated with mantle plume (Gibson, 2002). Sobolev et al. (2007) suggested that $\sim 2-30 \%$ of recycled crust is involved in the mantle melting to form MORBs, OIBs, continental basalts and komatiites. On the basis of phase relation and bulk rock composition of the Paraná-Etendeka ferropicrite, Tuff et al. (2005) speculated that ferropicrite primary melt is unlikely to have been derived from partial melt of normal peridotite and melting of garnet pyroxenite source may have been significant. The occurrence of garnet liquidus phase with clinopyroxene at pressure $\geq 6 \mathrm{GPa}$, leading Tuff et al. (2005) to propose garnet pyroxenite as mantle source for the Paraná- Etendeka ferropicrite. Ichiyama et al. (2006) have suggested that suitable ferropicrite sources include recycled ferrobasalts and $\mathrm{Fe}-\mathrm{Ti}$ gabbros (that should have been eclogitized in the source mantle). It is evident that ferropicrite liquid cannot originate in direct partial melting of ambient depleted peridotite (Stone et al., 1995).

Jakobsen et al. (2005) suggested a mixing between the peridotite-derived picritic melts with evolved Fe-rich basalts or immiscible liquids could result in a ferropicritic composition. However, liquid immiscibility and subsequent mixing process is unlikely cause for the generation of Ethiopian ferropicritic melt because of two reasons: (1) immiscibility tends to fade away with increasing pressure (Charlier and Grove, 2012); (2) such mixing process would be expected to produce significant disequilibrium textures, which are not observed among studied rocks.

The bulk rock $\mathrm{Cr} / \mathrm{Al}$ ratio (Fig. 8) may provide important clue about the source material. The extremely low bulk rock $\mathrm{Cr} / \mathrm{Al}$ ratio of Ethiopian ferropicrite and picritic ferrobasalt rocks evidence that they cannot be formed from the partial melting of only peridotite mantle source as the other mafic-ultramafic rocks (e.g., Sorachi-Yezo picrites, Japan; D-type Mikabu picrite, Japan; Pioneer komatiite, South Africa). Thus, we suggest that the significant contribution of recycled eclogitic material for the origin of Ethiopian ferropicrite and picritic ferrobasalts.

\section{Reversely zoned clinopyroxene; reaction with perido- titic mantle}

The origin of reversely zoned clinopyroxenes with resorbed texture has been attributed to magma mixing in many literatures (e.g., Wass, 1979; Simonetti et al., 1996). Kay (1978) suggested that the reversely zoned clinopyroxene may indicate reaction between a silicic melt and peridotite in the upper mantle. Reversely zoned clinopyroxenes have been also reported from Cenozoic basalts (Wang et al., 2007) and late Jurassic high-Mg adakaites of China (Gao et al., 2004), and suggested the presence of pyroxenite in the mantle source. Some of the analyzed clinopyroxene crystals of Ethiopian ferropicrite and picritic ferrobasalts exhibit unusually strong reverse zoning, which have a rim with distinctly high $\mathrm{Mg} \#, \mathrm{Cr}$ and Ni (Tables 4 and 5; Fig. 9). These zoning may indicate a significant increase of $\mathrm{Mg}$, $\mathrm{Cr}$ and $\mathrm{Ni}$ in the melt by assimilation of mantle peridotite (i.e., an olivine consuming reaction) or mixing with the peridotite-origin magma during magma ascent. Thus, the presence of these unusual reversely zoned clinopyroxene crystals may further proves the origin of Ethiopian ferropicrite and picritic ferrobasalt rocks from eclogitic source, and their reaction with the peridotitic mantle.

\section{Experimental constraints for the source lithology}

According to Yaxley and Green (1998), partial melting of relatively fusible eclogite inclusions produces $\mathrm{SiO}_{2}$-rich melts that react with olivine in the host garnet peridotite and generate a secondary garnet pyroxenite source in upwelling mantle. Progressive melting of this 're-fertilized' source may yields silica-undersaturated, more mafic par- 
tial melt. Gibson (2002) suggested this 're-fertilized' source model to ferropicrite petrogenisis. Herzberg (2011) also discussed about the reaction between eclogite (pyroxenite)-origin melt and the host peridotite for the generation OIB.

Partial melting experiment of equal proportion of mixed basalt (GA1, in the form of coesite eclogite) and peridotite (MPY90) at $1500{ }^{\circ} \mathrm{C}$ and $3.5 \mathrm{GPa}$ by Yaxley and Green (1998) and Yaxley (2000) yields a picritic melt that is close to Ethiopian ferropicrite composition. However, $\mathrm{GA} 1_{50} \mathrm{MPY} 90_{50}$ has slightly higher $\mathrm{MgO}(16.5$ wt $\%$ ) and $\mathrm{Al}_{2} \mathrm{O}_{3}$ (11.7 wt\%), lower $\mathrm{FeO}^{*}(12.50 \mathrm{wt} \%)$ as compared with Ethiopian ferropicrite (13.92 wt $\%$, $8.58 \mathrm{wt} \%$ and $14.00 \mathrm{wt} \%$, respectively) (Figs. 2a-2c). Tuff et al. (2005) proposed a source composition similar to a $75 \%$ basalt and $25 \%$ peridotite mixture at $\geq 5 \mathrm{GPa}$ for the Paraná-Etendeka ferropicrite, but it is not supported by experimental data at this pressure. Likewise, we suggest that a higher pressure mixture of eclogite-peridotite $\left(\mathrm{GA} 1_{75} \mathrm{MPY} 90_{25}\right)$ might be a plausible explanation to achieve the $\mathrm{FeO}^{*}$ content similar with Ethiopian ferropicrite.

The high $\mathrm{TiO}_{2}(>3 \mathrm{wt} \%)$ content of the Ethiopian ferropicrite and picritic ferrobasalts may further support the important role of Ti-rich recycled oceanic crust (eclogite).

\section{Hydrous mantle melting?}

The significance of water for the origin of ferropicrite is poorly known. However, different suggestions are given in literatures, such as the parental melt have been hydrous (Hanski and Smolkin, 1995, Fiorentini et al., 2008; their interpretation is based on the presence of kaersutite and phlogopite) and anhydrous (Gibson, 2002).

Partial melting experiment at high pressures (5-11 GPa) have been conducted on $\mathrm{H}_{2} \mathrm{O}$-saturated mantle peridotite (KLB-1) by Kawamoto and Holloway (1997). At about $7.5 \mathrm{GPa}$ the partial melt have lower $\mathrm{SiO}_{2}, \mathrm{TiO}_{2}$, $\mathrm{Al}_{2} \mathrm{O}_{3}, \mathrm{Na}_{2} \mathrm{O}$ and significantly higher $\mathrm{MgO}(32 \mathrm{wt} \%)$ than the Ethiopian ferropicrite, but similar $\mathrm{FeO}^{*}$. Thus, partial melt of $\mathrm{H}_{2} \mathrm{O}$ saturated peridotite unlikely to be the source of Ethiopian ferropicrite. Moreover, the absence of primary magmatic hydrous mineral phases as phenocrysts, in the groundmass or as inclusions from the studied Ethiopian ferropicrite precludes its derivation from hydrous magma source.

In summary, the presence of ferropicrite in Ethiopian LIP may provide additional evidence for the high temperature and high pressure mafic magmatism of Afar plume during Oligocene $(\sim 30 \mathrm{Ma})$ and thus it may further proves for the high eruption temperature in Phaner- ozoic time. The origin of these ferropicrite and picritic ferrobasalt rocks could be attributed to high pressure partial melting of mantle peridotite that possibly incorporated recycled components (eclogite or garnet pyroxenite).

\section{CONCLUSIONS}

The ferropicrite and picritic ferrobasalt from Lalibela area show high $\mathrm{Zr} / \mathrm{Y}$ and low $\mathrm{Al}_{2} \mathrm{O}_{3} / \mathrm{TiO}_{2}$ ratios indicating higher pressure melting or smaller degree of partial melting, which apparently contradicts to the high $\mathrm{Cr} \#$ of spinel in these rocks. The major and trace element data of the Ethiopian ferropicrite is compatible with the eclogitic source that possibly represents the recycled oceanic crust components in the upwelling Afar plume head. The occurrence of ferropicrite further supports the involvement of deep plume sources during Ethiopian LIP magmatism. In view of low $\mathrm{Cr} / \mathrm{Al}$ ratio of the bulk rock, the high $\mathrm{Cr} \#$ of spinel may be due to the very high temperature of the magma that originated in the eclogitic source. The occurrence of clinopyroxene crystals with extreme reverse zoning characteristics may further support the origin of Ethiopian ferropicrite and picritic ferrobasalts from eclogitic source at depths and their later interaction with the mantle peridotite.

\section{ACKNOWLEDGMENTS}

We are grateful to Tsuyoshi Miyamoto for his assistance with XRF measurement of whole-rock composition and to staffs of Kanazawa University for their comment and assistance with LA-ICP-MS trace element determinations. The third author acknowledges the financial support of Japan Society for Promotion of Science (JSPS) for his Bridge Fellow visiting to Tohoku University in January 2013 and the third author acknowledges the Grantin-Aid for Scientific research (C)-23540554 provided by the Ministry of Education, Culture, Sports, Science and Technology (MEXT), Japan. We also thank Hiroshi Kawabata and Jun-ichi Kimura for their constructive comments and suggestions that helped us to improve the manuscript. Masaaki Owada is thanked for the editorial work.

\section{REFERENCES}

Arai, S. (1994) Characterization of spinel peridotites by olivinespinel compositional relationships: review and interpretation. Chemical Geology, 113, 191-204.

Arndt, N., Lehnert, K. and Vasil'ev, Y. (1995) Meimechites: highly magnesian lithosphere-contaminated alkaline magmas from deep subcontinental mantle. Lithos, 34, 41-59.

Arndt, N.T., Naldrett, A.J. and Pyke, D.R. (1977) Komatiitic and 
Iron-rich tholeiitic lavas of Munro Township, Northeast Ontario. Journal of Petrology, 18, 319-369.

Arndt, N.T., Lesher, C.M. and Barnes, S.J. (2008) Komatiite. pp. 467, Cambridge University Press, Cambridge.

Ayalew, D., Barbey, P., Marty, B., Reisberg, L., Yirgu, G. and Pik, R. (2002) Source, genesis and timing of giant ignimbrite deposits associated with Ethiopian continental flood basalts. Geochimica et Cosmochimica Acta, 66, 1429-1448.

Ayalew, D. and Ishiwatari, A. (2011) Comparison of rhyolites from continental rift, continental arc and oceanic island arc: implication for the mechanism of silicic magma generation. Island Arc, 20, 78-93.

Barnes, S.J. (1985) The petrography and geochemistry of komatiite flows from the Abitibi Greenstone Belt and a model for their formation. Lithos, 18, 241-270.

Barnes, S.J. (1986) The distribution of chromium among orthopyroxene, spinel and silicate liquid at atmospheric pressure. Geochim. Cosmochim. Acta, 50, 1889-1909.

Beccaluva, L., Bianchini, G., Natali, C. and Siena, F. (2009) Continental flood basalts and mantle plumes: a case study of the northern Ethiopian Plateau. Journal of Petrology, 50, 13771403.

Charlier, B. and Grove, T.L. (2012) Experiments on liquid immiscibility along tholeiitic liquid lines of descent. Contributions to Mineralogy and Petrology, 164, 27-44.

Courtillot, V., Davaille, A., Besse, J. and Stock, J.M. (2003) Three distinct types of hotspots in the Earth's mantle. Earth and Planetary Science Letters, 205, 295-308.

Cox, K.G. and Jamieson, B.G. (1974) The olivine-rich lavas of Nuanetsi: a study of polybaric magmatic evolution. Journal of Petrology, 15, 269-301.

Dick, H.J.B. and Bullen, T. (1984) Chromium spinel as a petrogenetic indicator in abyssal and alpine-type peridotites and spatially associated lavas. Contributions to Mineralogy and Petrology, 86, 54-76.

Dietrich, V.J., Gansser, A., Sommerauer, J. and Cameron, W.E. (1981) Palaeogene komatiites from Gorgona Island, East Pacific: A primary magma for ocean floor basalts? Geochemical Journal, 15, 141-161.

Elkins-Tanton, L.T., Draper, D.S., Agee, C.B., Jewell, J., Thorpe, R.I. and Hess, P.C. (2007) The last lavas erupted during the main phase of the Siberian flood volcanic province: results from experimental petrology. Contributions to Mineralogy and Petrology, 153, 191-209.

Erdenesaihan, G., Ishiwatari, A., Orolmaa, D., Arai, S. and Tamura, A. (2013) Middle Paleozoic greenstons of the Hangay region, central Mongolia: Remnants of an accreted oceanic plateau and forearc magmatism. Journal of Mineralogical and Petrological Sciences, 108, 303-325.

Fabriès, J. (1979) Spinel-olivine geothermometry in peridotites from ultramafic complexes. Contributions to Mineralogy and Petrology, 69, 329-336.

Fiorentini, M.L., Beresford, S.W., Deloule, E., Hanski, E., Stone, W.E. and Pearson, N.J. (2008) The role of mantle-derived volatiles in the petrogenesis of Palaeoproterozoic ferropicrites in the Pechenga Greenstone Belt, northwestern Russia: Insights from in-situ microbeam and nanobeam analysis of hydromagmatic amphibole. Earth and Planetary Science Letters, 268, 2-14.

Francis, D., Ludden, J., Johnstone, R. and Davis, W. (1999) Picrite evidence for more Fe in Archean mantle reservoirs. Earth and Planetary Science Letters, 167, 197- 213.
Gao, S., Rudnick, R.L., Yuan, H.L., Liu, X.M., Liu, Y.S., Xu, W.L., Ling, W.L., Ayers, J., Wang, X.C. and Wang, Q.H. (2004) Recycling lower continental crust in the North China craton. Nature, 432, 892-897.

George, R., Rogers, N. and Kelley, S. (1998) Earliest magmatism in Ethiopia: evidence for two mantle plumes in one flood basalt province. Geology, 26, 923-926.

Gibson, S.A. (2002) Major element heterogeneity in Archean to Recent mantle plume starting-heads. Earth and Planetary Science Letters, 195, 59-74.

Gibson, S.A., Thompson, R.N. and Dickin, A.P. (2000) Ferropicrites: geochemical evidence for Fe-rich streaks in upwelling mantle plumes. Earth and Planetary Science Letters, 174, 355374.

Goldstein, S.B. and Francis, D.M. (2008) The Petrogenesis and mantle source of Archaean ferropicrites from the Western $\mathrm{Su}$ perior Province, Ontario, Canada. Journal of Petrology, 49, 1729-1753.

Gurenko, A.A., Hansteen, T.H. and Schmincke, H.U. (1996) Evolution of parental magmas of Miocene shield basalts of Gran Canaria (Canary Islands): constraints from crystal, melt and fluid inclusions in minerals. Contributions to Mineralogy and Petrology, 89, 422-435.

Hanski, E. (1992) Petrology of the Pechenga ferropicrites and cogenetic, Ni-bearing gabbro-wehrlite intrusions, Kola Peninsula Russia. Bulletin of the Geological Survey of Finland, 367, 192.

Hanski, E.J. and Smolkin, V.F. (1989) Pechenga ferropicrites and other early Proterozoic picrites in the eastern part of the Baltic Shield. Precambrian Research, 45, 63-82.

Hanski, E.J. and Smolkin, V.F. (1995) Iron- and LREE-enriched mantle source for early Proterozoic intraplate magmatism as exemplified by the Pechenga ferropicrites, Kola Peninsula, Russia. Lithos, 34, 107-125.

Heinonen, J.S. and Luttinen, A.V. (2008) Jurassic dikes of Vestfjella, Western Dronning Maud Land, Antarctica: geochemical tracing of ferropicrite sources. Lithos, 105, 347-364.

Heinonen, J.S. and Luttinen, A.V. (2010) Mineral chemical evidence for extremely magnesian subalkaline melts from the Antarctic extension of the Karoo large igneous province. Contributions to Mineralogy and Petrology, 99, 201-217.

Herzberg, C. (2011) Identification of source lithology in the Hawaiian and Canary Islands: implications for origins. Journal of Petrology, 52, 113-146.

Herzberg, C. and Asimow, P.D. (2008) Petrology of some oceanic island basalts: PRIMELT2.XLS software for primary magma calculation. Geochemistry Geophysics Geosystems, 8, doi: 10.1029GC002057.

Hofmann, C., Courtillot, V., Feraud, G., Rochette, P., Yirgu, G., Ketefo, E. and Pik, R. (1997) Timing of the Ethiopian flood basalt event and implications for plume birth and global change. Nature, 389, 838-841.

Ichiyama, Y., Ishiwatari, A., Hirahara, Y. and Shuto, K. (2006) Geochemical and isotopic constraints on the genesis of the Permian ferropicritic rocks from the Mino-Tamba belt, SW Japan. Lithos, 89, 47-65.

Ichiyama, Y., Ishiwatari, A., Kimura, J., Senda, R., Kawabata, H. and Tatsumi, Y. (2012) Picrites in central Hokkaido: Evidence of extremely high temperature magmatism in the Late Jurassic ocean recorded in an accreted oceanic plateau. Geology, 40, 411-414.

Ichiyama, Y., Ishiwatari, A., Kimura, J.-I., Senda, R. and Miya- 
moto, T. (2014) Jurassic plume-origin ophiolites in Japan: accreted fragments of oceanic plateaus. Contributions to Mineralogy and Petrology, doi:10.1007/s00410-014-1019-1.

Ishida, H., Morishita, T., Arai, S. and Shirasaka, M. (2004) Simultaneous in-situ multi-element analysis of minerals on thin section using LA-ICP-MS. Science Report of Kanazawa University, 48, 31-42.

Ishiwatari, A. (1985) Igneous petrogenesis of the Yakuno ophiolite (Japan) in the context of the diversity of ophiolites. Contributions to Mineralogy and Petrology, 89, 155-167.

Ishiwatari, A. and Imasaka, M. (2002) Picritic basalt from the Miocene Yoka Formation in the Tango Peninsula, Kyoto Prefecture, south-western Japan. The Journal of the Geological Society of Japan, 108, 671-684 (In Japanese with English abstract).

Jakobsen, J.K., Veksler, I.V., Tegner, C. and Brooks, C.K. (2005) Immiscible iron- and silica-rich melts in basalt petrogenesis documented in Skaergaard intrusion. Geology, 33, 885-888.

Kamenetsky, V.S., Crawford, A.J. and Meffre, S. (2001) Factors controlling chemistry of magmatic spinel: an empirical study of associated olivine, $\mathrm{Cr}$-spinel and melt inclusions from primitive rocks. Journal of Petrology, 42, 655-671.

Kamenetsky, V.S., Chung, S., Kamenetsky, M.B. and Kuzmin, D.V. (2012) Picrites from the Emeishan large igneous province, SW China: a compositional continuum in primitive magmas and their respective mantle sources. Journal of Petrology, 53, 2095-2113.

Kawamoto, T. and Holloway, J.R. (1997) Melting temperature and partial melt chemistry of $\mathrm{H}_{2} \mathrm{O}$-saturated mantle peridotite to 11 Giga Pascals. Science, 276, 240-243.

Kawabata, H., Hanyu, T., Chang, Q., Kimura, J., Nichols, A.R.L. and Tatsumi, Y. (2011) The Petrology and Geochemistry of St. Helena Alkali Basalts: Evaluation of the Oceanic Crustrecycling Model for HIMU OIB. Journal of Petrology, 52, $791-838$

Kay, R.W. (1978) Aleutian magnesian andesites: melt from subducted Pacific Ocean crust. Journal of Volcanology and Geothermal Research, 4, 117-132.

Kieffer, B., Arndt, N., Lapierre, H., Bastien, F., Bosch, D., Pecher, A., Yirgu, G., Ayalew, D., Weis, D., Jerram, D.A., Keller, F. and Meugniot, C. (2004) Flood and shield basalts from Ethiopia: magmas from the African superswell. Journal of Petrology, 45, 793-834.

Kogiso, T. and Hirschmann, M.M. (2001) Experimental study of clinopyroxenite partial melting and the origin of ultra-calcic melt inclusions. Contributions to Mineralogy and Petrology, $142,347-360$.

Kogiso, T., Hirschmann, M.M. and Frost, D.J. (2003) High pressure partial melting of garnet pyroxenite: possible mafic lithologies in the source of ocean island basalts. Earth and Planetary Science Letters, 216, 603-617.

Kogiso, T., Hirschmann, M.M. and Pertermann, M. (2004) Highpressure partial melting of mafic lithologies in the mantle. Journal of Petrology, 45, 2407-2422.

Larsen, L.M. and Pedersen, A.K. (2000) Processes in high-Mg, high-T magmas: evidence from olivine, chromite and glass in Palaeogene picrites from West Greenland. Journal of Petrology, 41, 1071-1098.

Le Bas, M.J. (2000) IUGS reclassification of the high-Mg and picritic volcanic rocks. Journal of Petrology, 41, 1467-1470.

Liu, Y., Gao, S., Kelemen, P.B. and Xu, W. (2008) Recycled crust controls contrasting source compositions of Mesozoic and
Cenozoic basalts in the North China Craton. Geochimica et Cosmochimica Acta, 72, 2349-2376.

López, J.C. and Ishiwatari, A. (2002) Petrogenesis of tholeiitic basalt, calc-alkaline basaltic andesite and high magnesian andesite lava succession of the Oligo-Miocene Anamizu Formation in northeastern Noto Peninsula, central Japan. Journal of Mineralogical and Petrological Sciences, 97, 85-113, [Errata in 98, 165 (2003)].

Marty, B., Pik, R. and Gezahegn, Y. (1996) Helium isotopic variations in Ethiopian plume lavas: nature of magmatic sources and limit on lower mantle contribution. Earth and Planetary Science Letters, 144, 223-237.

McDonough, W.F. and Sun, S.-s. (1995) The composition of the Earth. Chemical Geology, 120, 223-254.

McKenzie, D. and Bickle, M.J. (1988) The volume and composition of melt generated by extension of the lithosphere. Journal of Petrology, 29, 625-679.

Mohr, P. and Zanettin, B. (1988) The Ethiopian flood basalt province. In Continental Flood Basalts (MacDougall, J.D. Ed.). Kluwer, Dordrecht, 63-110.

Natali, C., Beccaluva, L., Bianchini, G. and Siena, F. (2011) Rhyolites associated to Ethiopian CFB: Clues for initial rifting at Afar plume axis. Earth and Planetary Science Letters, 312, 59-68.

Parman, S.W. and Grove, T.L. (2004) Harzburgite melting with and without $\mathrm{H}_{2} \mathrm{O}$ : experimental data and predictive modeling. Journal of Geophysical Research, 109 (B2). doi:10.1029/ 2003JB002566.

Pearce, N.J.G., Perkins, W.T., Westgate, J.A., Gorton, M.P., Jackson, S.E., Neal, C.R. and Chenery, S.P. (1997) A compilation of new and published major and trace element data for NIST SRM 610 and NIST SRM 612 glass reference materials. Geostandard Newsletter, 21, 115-144.

Pedersen, A.K. (1985) Reaction between picrite magma and continental crust: early Tertiary silicic basalts and magnesian andesites from Disko, West Greenland. Bulltin Gronlands Geologiske Undersogelse, 152, 126.

Pertermann, M. and Hirschmann, M.M. (2003) Anhydrous partial melting experiments on MORB-like eclogite: phase relations, phase compositions and mineral-melt partitioning of major elements at 2-3 GPa. Journal of Petrology, 44, 2173-2201.

Piccirillo, E.M., Justin Visentin, E., Zanettin, B., Joron, J.K. and Treuil, M. (1979) Geodynamic evolution from plateau to rift: Major and trace element geochemistry of the central eastern Ethiopian plateau volcanics. Neues Jahrbuch für Geologie und Paläontologie, 158, 139-179.

Pik, R., Deniel, C., Coulon, C., Yirgu, G., Hofmann, C. and Ayalew, D. (1998) The northwestern Ethiopian Plateau flood basalts: Classification and spatial distribution of magma types. Journal of Volcanology and Geothermal Research, 81, 91111.

Putirka, K.D. (2005) Mantle potential temperatures at Hawaii, Iceland, and the mid-ocean ridge system, as inferred from olivine phenocrysts; evidence for thermally driven mantle plumes. Geochemistry, Geophysics, Geosystems, 6, doi:10.1029/ 2005GC000915.

Putirka, K.D. (2008) Thermometers and barometers for volcanic systems. Minerals, inclusions and volcanic processes. In Reviews in Mineralogy and Geochemistry (Putirka, K.D. and Tepley III, F.J. Eds.). 69. Mineralogical Society of America, 61-120.

Riley, T.R., Leat, P.T., Curtis, M.L., Millar, I.L., Duncan, R.A. and 
Fazel, A. (2005) Early-middle Jurassic dolerite dykes from western Dronning Maud Land (Antarctica): identifying mantle sources in the Karoo large igneous province. Journal of Petrology, 46, 1489-1524.

Ritsema, J., Van Heijst, H.J. and Woodhouse, J.H. (1999) Complex shear wave velocity structure imaged beneath Africa and Iceland. Science, 286, 1925-1928.

Robin-Popieul, C.C.M., Arndt, N.T., Chauvel, C., Byerly, G.R., Sobolev, A.V. and Wilson, A. (2012) A New Model for Barberton Komatiites: Deep Critical Melting with High Melt Retention. Journal of Petrology, 53, 2191-2229.

Rochette, P., Tamrat, E., Feraud, G., Pik, R., Courtillot, V., Ketefo, E., Coulon, C., Hoffmann, C., Vandamme, D. and Yirgu, G. (1998) Magnetostratigraphy of the Ethiopian traps. Earth and Planetary Science Letters, 164, 497-510.

Rogers, N.W., Davies, M.K., Parkinson, I.J. and Yirgu, G. (2010) Osmium isotopes and $\mathrm{Fe} / \mathrm{Mn}$ ratios in Ti-rich picritic basalts from the Ethiopian flood basalt province. No evidence from core contribution to the Afar plume. Earth Planetary Science Letters, 296, 413-422.

Sigurdsson, H. and Schilling, J.G. (1976) Spinels in Mid-Atlantic Ridge basalts: chemistry and occurrence. Earth and Planetary Science Letters, 29, 7-20.

Sigurdsson, I.A., Steinthorsson, S. and Grönvold, K. (2000) Calcium-rich melt inclusions in $\mathrm{Cr}$ spinels from Borgarhraun, northern Iceland. Earth and Planetary Science Letters, 183, 15-26.

Simkin, T. and Smith, J.V. (1970) Minor-element distribution in olivine. Journal of Geology, 78, 304-325.

Simonetti, A., Shore, M. and Bell, K. (1996) Diopside phenocrysts from lavas, Napak Volcano, Eastern Uganda: Evidence for magma mixing. Canadian Mineralogist, 34, 411-421.

Sobolev, A.V. and Danyushevsky, L.V. (1994) Petrology and geochemistry of boninites from the north termination of the Tonga Trench: Constraints on the generation conditions of primary high-Ca boninite magmas. Journal of Petrology, 35, 1183-1211.

Sobolev, A.V., Hofmann, A.W., Kuzmin, D.V., Yaxley, G.M., Anderson, A.T., Arndt, N.T., Chung, S.-L., Garcia, M.O., Gurenko, A.A., Danyushevsky, L.V., Elliott, T., Frey, F.A., Kamenetsky, V.S., Kerr, A.C., Krivolutskaya, N.A., Matvienkov, V.V., Nikogosian, I.K., Rocholl, A., Sigurdsson, I., Suschevskaya, N.M. and Teklay, M. (2007) The amount of recycled crust in sources of mantle-derived melts. Science, 316, 412417.

Stiegler, M.T., Cooper, M., Byerly, G.R. and Lowe, D.R. (2012) Geochemistry and petrology of komatiites of the Pioneer U1tramafic Complex of the 3.3 Ga Weltevreden Formation, Barberton greenstone belt, South Africa. Precambrian Research, 212-213, 1-12.

Stone, W.E., Crocket, J.H., Dickin, A.P. and Fleet, M.E. (1995)
Origin of Archean ferropicrites: geochemical constraints from the Boston Creek Flow, Abitibi greenstone belt, Ontario, Canada. Chemical Geology, 121, 51-71.

Thompson, R.N. and Gibson, S.A. (2000) Transient high temperatures in mantle plume heads inferred from magnesian olivines in Phanerozoic picrites. Nature, 407, 502-506.

Tuff, J., Takahashi, E. and Gibson, S.A. (2005) Experimental constraints on the role of garnet pyroxenite in the genesis of highFe mantle plume derived melts. Journal of Petrology, 46, 2023-2058.

Tuff, J. and Gibson, S.A. (2007) Trace-element partitioning between garnet, clinopyroxene and Fe-rich picritic melts at 3 to $7 \mathrm{GPa}$. Contributions to Mineralogy and Petrology, 153, 369-387.

Walter, M.J. (1998) Melting of garnet peridotite and the origin of komatiite and depleted lithosphere. Journal of Petrology, 39, 29-60.

Wang, W., Xu, W., Wang, D., Ji, W., Yang, D. and Pei, F. (2007) Caiyuanzi Paleogene basalts and deep-derived xenocrysts in eastern Liaoning, China: constraints on nature and deep process of the Cenozoic lithospheric mantle. Journal of Mineralogy and Petrology, 27, 63-70.

Wass, S.Y. (1979) Multiple origins of clinopyroxene in alkalic basaltic rocks. Lithos, 12, 115-132.

Wilkinson, J.F.G. and Hensel, H.D. (1988) The petrology of some picrites from Mauna Loa and Kilauea volcanoes, Hawaii. Contributions to Mineralogy and Petrology, 98, 326-345.

Wooden, J.L., Czamanske, G.K., Fedorenko, V.A., Arndt, N.T., Chauvel, C., Bouse, R.M., King, W.B.S., Knight, R.J. and Siems, D.F. (1993) Isotopic and trace-element constraints on mantle and crustal contributions to Siberian continental flood basalts, Noril'sk area, Siberia. Geochimica et Cosmochimica Acta, 57, 3677-3704.

Yaxley, G.M. (2000) Experimental study of the phase and melting relations of homogeneous basalt + peridotite mixtures and implications for the petrogenesis of flood basalts. Contributions to Mineralogy and Petrology, 139, 326-338.

Yaxley, G.M. and Green, D.H. (1998) Reactions between eclogite and peridotite: mantle refertilisation by subduction of oceanic crust. Schweizerische mineralogische und petrographische Mitteilungen, 78, 243-255.

Zhang, Z., Mahoney, J.J., Mao, J. and Wang, F. (2006) Geochemistry of picritic and associated basalt flows of the western Emeishan flood basalt province, China. Journal of Petrology, 47, 1997-2019.

Manuscript received November 25, 2013

Manuscript accepted June 25, 2014

Published online August 2, 2014

Manuscript handled by Masaaki Owada 